\title{
COVERS IN FREE LATTICES
}

BY

RALPH FREESE AND J. B. NATION ${ }^{1}$

\begin{abstract}
In this paper we study the covering relation $(u \succ v)$ in finitely generated free lattices. The basic result is an algorithm which, given an element $w \in \operatorname{FL}(X)$, finds all the elements which cover or are covered by $w$ (if any such elements exist). Using this, it is shown that covering chains in free lattices have at most five elements; in fact, all but finitely many covering chains in each free lattice contain at most three elements. Similarly, all finite intervals in $\operatorname{FL}(X)$ are classified; again, with finitely many exceptions, they are all one-, two- or three-element chains.
\end{abstract}

Introduction. This paper was motivated by two questions about covers in free lattices. (1) For a lattice term $w$ with $n$ variables, can one recursively decide if (the element of $\operatorname{FL}(n)$ corresponding to) $w$ covers any element in $\operatorname{FL}(n)$ ? (2) Is there an element $w$ in FL(3) which neither covers nor is covered by any element of FL(3)? The first problem was suggested to us by Q. F. Stout and the second by David Kelly.

Covers in free lattices are an interesting and important part of lattice theory. Lattices of the form $\operatorname{FL}(n) / \psi$, where $\psi$ is the unique maximal congruence separating $u$ from $v$ when $u$ covers $v$ in $\operatorname{FL}(n)$, are called splitting lattices. These lattices and the corresponding coverings play an especially important role in the equational theory of lattices; see McKenzie's paper [14].

Some of the best results on covers are: Ralph McKenzie's theorem that one can recursively decide if $u$ covers $v$ for lattice terms $u$ and $v$ [14]; Alan Day's theorem that $\mathrm{FL}(n)$ is weakly atomic (every proper interval contains a covering) [3]; R. A. Dean's unpublished result that there are elements of FL(3) which do not cover any element.

In this paper we answer questions (1) and (2) in the affirmative. We show that in the first problem we may assume $w$ is join irreducible. In fact we show that $w$ has a lower cover if and only if one of its canonical joinands does. We then show that there is a bound on the complexity of the lower cover of $w$ in terms of the complexity of $w$. Thus there are only finitely many candidates for the lower cover. This together with McKenzie's algorithm gives an algorithm for testing if $w$ has a lower cover.

Unfortunately, this algorithm is difficult to apply and we were unable to use it to solve (2). Part of McKenzie's algorithm takes the generating set $X$ of the free lattice

Received by the editors June 14, 1983 and, in revised form, March 15, 1984.

1980 Mathematics Subject Classification. Primary 06B25.Received by the editors June 14, 1983 and, in revised form, March 15, 1984.

${ }^{1}$ This research was supported by NSF Grants MCS80-02311A1 (Freese) and MCS81-01655 (Nation). 
$\mathrm{FL}(X)$ and alternately closes under joins and meets a finite number of times. The result is a finite lattice and the procedure then asks certain questions about the homomorphic images of this lattice. Unfortunately even if $|X|=3$, the first nontrivial case, and we close under joins and meets each twice, the lattice has 677 elements.

We introduce a modification of this procedure. To each join irreducible $w \in \operatorname{FL}(n)$ we associate a set $J(w)$ of join irreducible elements of $\operatorname{FL}(n)$. These elements correspond (in a specified way) to certain subterms of the term representing $w$. Then we close $J(w)$ under joins and add a zero. The result is a finite lattice $L(w)$. Then $w$ will have a lower cover if and only if $L(w)$ is semidistributive. Even for moderately complex terms $L(w)$ is reasonably small. $L(w)$ has many nice properties which are given in $\$ \S 3$ and 4. In particular, if $u \in J(w)$, then $L(u)$ is a homomorphic image of $L(w)$. It follows that if $u$ has no lower cover then neither does $w$. From this and dual considerations it is easy to find elements in $\mathrm{FL}(3)$ which have neither upper nor lower covers. In fact, $(x(y+z)+y z)(y(x+z)+x z)$ is such a word. In $\S 4$ we give a table of some elements of FL(3) and their upper and lower covers.

$\$ 4$ also gives purely syntactical algorithms for testing if $w$ has a lower cover and finding it if it does. These algorithms are efficient and we have implemented them on a microcomputer using muLISP, a version of LISP for microcomputers. From our algorithm we are able to show that if $w$ is join irreducible and has a lower cover then for each canonical meetand $v$ of $w$ all but exactly one of the canonical summands of $v$ is below $w$. At the end of the section a theorem which relates the canonical forms of a completely join irreducible element and its lower cover is given.

In $\$ 5$ we give a syntactic proof of Day's theorem. Day's original proof is based on his doubling construction. This doubling construction is also in our proof, although it is hidden. Given $u>v$ in $\operatorname{FL}(X), X$ finite, our proof effectively finds elements $s$ covering $t$ with $u \geqslant s>t \geqslant v$. Moreover we show that such $s$ and $t$ can be found whose complexity is bounded by the sum of the complexities of $u$ and $v$. Examples are given to show this bound is the best possible.

Bjarni Jónsson suggested another interesting question: Is there a bound on the length of covering chains $a_{0} \succ a_{1} \succ \cdots>a_{k}$ in $\operatorname{FL}(X)$ [19]? The longest covering chains we knew of were five-element chains at the top and bottom of FL(3), and four-element chains at the top and bottom of $\operatorname{FL}(n)$ when $n \geqslant 4(\S 6)$.

This investigation led directly to a question which is interesting in its own right. Call an element $w \in \operatorname{FL}(X)$ totally atomic if, whenever $u>w$, there exists $v$ with $u \geqslant v \succ w$, and the dual property holds. What are the totally atomic elements in $\mathrm{FL}(X)$ ? In $\$ 7$ we show that the totally atomic elements in $\mathrm{FL}(X)$ are precisely the elements having a certain simple form. In particular, each $\operatorname{FL}(n)(n \in \omega)$ contains only finitely many totally atomic elements. $\S 8$ contains several technical lemmas relating totally atomic elements to Jónsson's question. The principal connection is this: If $a_{0}>a_{1}>a_{2}$ in $\operatorname{FL}(X)$ and $a_{1}$ is, say, meet irreducible, then the (unique) member of the canonical join representation of $a_{1}$ which is not below $a_{2}$ must be totally atomic.

Using this, we show in $\S 9$ that chains of covers in free lattices can have length at most 4 and these only occur at the top and bottom of FL(3). Chains of length 3 also 
only occur at the top and bottom of $\operatorname{FL}(n)$. We show, however, that there are infinitely many chains of covers of length 2 in $\mathrm{FL}(4)$. We also classify all finite interval sublattices of free lattices. In $\mathrm{FL}(3)$ the interval $x y+x z+y z / x y z$ is finite. Every finite interval sublattice of a free lattice is isomorphic or dually isomorphic to a sublattice of $x y+x z+y z / x y z$. Moreover, the only finite interval sublattices of FL $(n)$ which occur infinitely often are the one-, two- and three-element chains.

We conclude with some remarks on arbitrary intervals in $\operatorname{FL}(X)$. In particular, we prove that every interval $u / v$ in a free lattice is isomorphic to a projective lattice which is generated by its doubly (meet and join) irreducible elements.

1. Preliminaries. We recall here some of the terminology and results which we will be using. We say that $a$ covers $b$ in a lattice if $a>b$ and there is no element $c$ with $a>c>b$. We write $a>b$. By a covering pair we mean a pair of elements $a, b$ with $a \succ b$. If $a \succ b$, then $a$ is called an upper cover of $b$, and $b$ is a lower cover of $a$.

We are concerned with finding covers of elements in free lattices. If $X$ is infinite, then $\mathrm{FL}(X)$ has no coverings. On the other hand, Day's result [3] shows that finitely generated free lattices have many coverings. Our problem will be to find upper or lower covers, if there are any, for a given element in a fixed finitely generated free lattice $\mathrm{FL}(n)$.

Let $J(L)$ denote the set of all nonzero join irreducible elements in a lattice $L$, and $M(L)$ the set of all nonunit meet irreducible elements. For technical reasons related to the fact that 0 has no lower cover, we exclude 0 from $J(L)$ and often when we refer to a join irredicible element of $L$ we tacitly assume it is not 0 . If $w \in J(\operatorname{FL}(X))$, then $w$ covers at most one element, which, if it exists, will be denoted by $w_{*}$. If $w \in J(\mathrm{FL}(X))$ has a lower cover, then $w$ is completely join irreducible in that $w$ is not the supremum of any subset of $\operatorname{FL}(X)$ not containing $w$. The converse is also obviously true and thus we shall use the terminology " $w$ has a lower cover" and " $w$ is completely join irreducible" interchangeably. If $w_{*}$ exists, then there is a unique maximal element $v \in \mathrm{FL}(X)$ such that $v \geqslant w_{*}$ but $v \neq w$ [14]; this element will be denoted by $\kappa(w)$. In the proof of Theorem 2.1 we will indicate how to find $\kappa(w)$ when it exists.

If $L$ is a finite lattice and $p \in J(L)$, then $p$ has a lower cover $p_{*}$, and there may or may not exist a unique element $\kappa_{L}(p)$ which is maximal with respect to being above $p_{*}$ but not above $p$. In fact, we note that for a finite lattice $L, \kappa_{L}(p)$ exists for every $p \in J(L)$ if and only if $L$ satisfies

$$
u=a b=a c \text { implies } u=a(b+c) .
$$

To see this, first assume that $L$ satisfies $\left(\mathrm{SD}_{\wedge}\right)$ and let $p \in J(L)$. If $K=\{x \in L$ : $\left.p x=p_{*}\right\}$, then $\left(\mathrm{SD}_{\wedge}\right)$ implies $\sum K \in K$, so that $\kappa_{L}(p)=\sum K$. On the other hand, assume that $L$ fails ( $\mathrm{SD}_{\wedge}$ ) with $u=a b=a c<a(b+c)$. Let $q$ be an element of $L$ which is minimal with respect to $q \leqslant a(b+c)$ but $q \nless u$. Clearly $q \in J(L)$ and $q_{*} \leqslant u$. Using $q \leqslant a$, we calculate $q b=q c=q_{*}$ whereas $q(b+c)=q$, which means that $\kappa_{L}(q)$ does not exist.

The dual of $\left(\mathrm{SD}_{\wedge}\right)$ is

$$
v=a+b=a+c \text { implies } v=a+b c .
$$


A lattice which satisfies both $\left(\mathrm{SD}_{\wedge}\right)$ and $\left(\mathrm{SD}_{\vee}\right)$ is called semidistributive.

A finitely generated lattice $L$ is lower bounded if for every homomorphism $f$ : $\operatorname{FL}(X) \rightarrow L$ (where $X$ is finite) and every $a \in L,\{u \in \operatorname{FL}(X): f(u) \geqslant a\}$ either is empty or has a least element. Equivalently, $L$ is lower bounded if there exists an epimorphism $f: \operatorname{FL}(X) \rightarrow L$ such that every $a \in L$ has a least preimage. (See [14, Lemma 5.4, or 13, Theorem 4.2].) Upper bounded is defined dually, and $L$ is bounded if it is both lower al sper bounded.

For nonempty fin, Ibsets $U$ and $V$ of a lattice $L$, we say that $U$ refines $V$ if for every $u \in U$ there exists $v \in V$ such that $u \leqslant v$. We write $U \ll V$ for this. $U \gg V$ is defined dually: for all $u \in U$ there is a $v \in V$ with $u \geqslant v$. We call $V$ a join-cover of $a \in L$ if $a \leqslant \sum V$. A join-cover $V$ is nontrivial if $a \nless v$ for each $v \in V$. $V$ is a minimal join-cover of $a$ if whenever $a \leqslant \sum U$ and $U$ refines $V$, then $V \subseteq U$.

We will now describe a particularly simple algorithm, due to Bjarni Jónsson, for determining whether a finitely generated lattice is lower bounded. Let $D_{0}(L)$ denote the set of join prime elements of $L$, i.e., those elements which have no nontrivial join-cover. For $k>0$, let $a \in D_{k}(L)$ if every nontrivial join-cover $V$ of $a$ has a refinement $U \subseteq D_{k-1}(L)$ which is also a join-cover of $a$. Then a finitely generated lattice $L$ is lower bounded if and only if $\bigcup_{k \geqslant 0} D_{k}(L)=L$. (We will sketch one direction of the proof below; for the converse and more details, see [13].)

Observe that, from the definition, $D_{0}(L) \subseteq D_{1}(L) \subseteq D_{2}(L) \subseteq \cdots$. If $L$ is lower bounded and $a \in L$, we define the $D$-rank $\rho(a)$ to be the least integer $k$ such that $a \in D_{k}(L)$. It is easy to see that if $U$ is a finite nonempty subset of $D_{k}(L)$, then $\sum U \in D_{k+1}(L)$. Thus a finite lattice $L$ will be lower bounded if and only if $J(L) \subseteq D_{n}(L)$ for some $n$.

To test for upper boundedness, we define $D_{k}^{\prime}(L)$ and the $D^{\prime}$-rank $\rho^{\prime}(a)$ dually to the above.

Let $L$ be a finitely generated lattice, and let $f: \operatorname{FL}(X) \rightarrow L$ (with $X$ finite) be an epimorphism. If $L$ is lower bounded, then for $a \in L$ we let $\beta_{f}(a)$ denote the least preimage of $a$. If $L$ is upper bounded, then $\alpha_{f}(a)$ denotes the greatest preimage of $a$. The subscripts will be omitted when there is no danger of confusion. The proof that Jónsson's algorithm works in fact tells us how to find $\beta(a)$. Assume $\bigcup_{k \geqslant 0} D_{k}(L)=L$. For all $a \in L$, define

$$
\beta_{0}(a)=\prod\{x \in X: f(x) \geqslant a\} .
$$

If $a \in D_{0}(L)$, it is not hard to see that $\beta(a)=\beta_{0}(a)$. Assume we have found $\beta(b)$ for all $b \in D_{k}(L)$, and let $a \in D_{k+1}(L)$. Let $C(a)$ denote the set of all minimal nontrivial join-covers of $a$. By the definition of $D_{k+1}(L)$, we have $U \subseteq D_{k}(L)$ whenever $U \in C(a)$. By Lemma 3.1 of [13], $D_{k}(L)$ is finite, so $C(a)$ is finite. Also, every nontrivial join-cover of $a$ in $L$ refines to a join-cover in $C(a)$. Let

$$
\beta(a)=\beta_{0}(a) \prod_{U \in C(a)} \sum_{b \in U} \beta(b)
$$

The reader can now check that for all $w \in \operatorname{FL}(X), f(w) \geqslant a$ if and only if $w \geqslant \beta(a)$. Since $f$ is onto, this means that $\beta(a)$, as defined above, is the least preimage of $a$. Finally, since we are assuming that $\bigcup_{k \geqslant 0} D_{k}(L)=L$, this process inductively defines $\beta(a)$ for every $a \in L$, which shows that $L$ is lower bounded. 
If $L$ is a finite, lower bounded lattice and $f: \operatorname{FL}(X) \rightarrow L$ (with $X$ finite) is an epimorphism, then $\beta_{f}$ is a join-preserving embedding of $L$ into $\operatorname{FL}(X)$. In particular, it suffices to compute $\beta(a)$ only for all $a \in J(L)$, and in practice this is what we shall do.

If $a>b$ in a lattice $L$, then by Dilworth's characterization of lattice congruences there is a unique congruence $\psi_{a b}$ on $L$ which is maximal with the property $(a, b) \notin \psi_{a b}$. One of the main results of McKenzie [14] is that if $u \succ v$ in $\operatorname{FL}(n)$, then $\operatorname{FL}(n) / \psi_{u v}$ is a finite, subdirectly irreducible, bounded lattice. (We will give a new proof of this in §4.) Lattices with these properties are called splitting lattices. Conversely, every splitting lattice is isomorphic to $\operatorname{FL}(n) / \psi_{u v}$ for some covering pair in a free lattice.

Free lattices satisfy the following lattice condition due to Whitman [17]:

(W) $a=\prod_{i=1}^{n} a_{i} \leqslant \sum_{j=1}^{m} b_{j}=b$ implies there is an $i$ with $a_{i} \leqslant b$ or a $j$ with $a \leqslant b_{j}$.

Whitman's solution to the word problem also implies that every element $u$ of a free lattice can be represented by a term of minimal length which is unique up to commutivity and associativity. This term is either a variable or join or a meet of simpler terms. If it is a join of simpler terms, none of which is formally a join, then the elements of the free lattice corresponding to these terms are called the canonical joinands of $u$ and denoted $\operatorname{CJ}(u)$. In the other case, $w$ is join irreducible and $\mathrm{CJ}(u)=\{u\}$. We shall repeatedly use the following fact which semantically defines $\mathrm{CJ}(u)$ : if $\mathrm{CJ}(u)=U$ and $u=\sum V$, then $U$ refines $V$ [17]. Canonical meetands are of course defined dually and denoted by $\operatorname{CM}(u)$. We will use the phrase " $u=u_{1} \cdots u_{m}$ canonically" to mean that $\left\{u_{1}, \ldots, u_{m}\right\}$ are the canonical meetands of $u$. We will also say $u_{1} \cdots u_{m}$ is in canonical form.

Whitman has a simple algorithm to test if a lattice term is in canonical form. The corresponding semantical statement for elements of $\operatorname{FL}(X)$ is this: if $w=w_{1} \cdots w_{n}$ in $\mathrm{FL}(X)$, where each $w_{i}$ is meet irreducible, then $w_{1}, \ldots, w_{n}$ are the canonical meetands of $w$ if and only if $\left\{w_{1}, \ldots, w_{n}\right\}$ is an antichain and if $u \in \operatorname{CJ}\left(w_{i}\right)$, then $u \neq w$, $i=1, \ldots, n$.

2. Decidability. We are looking for an algorithm which, given a lattice term $t\left(x_{1}, \ldots, x_{n}\right)$, determines recursively whether the element $w \in \operatorname{FL}(n)$ corresponding to $t$ has a lower cover. If $w$ is joint irreducible in $\operatorname{FL}(n)$, then $w$ has a lower cover if and only if it is completely join irreducible. If $w$ has a lower cover $w_{*}$ then, as mentioned above, there is a unique largest element $\kappa(w)$ above $w_{*}$ but not above $w$. It is easy to see that $\kappa(w)$ is completely meet irreducible with unique upper cover $\kappa(w)^{*}=\kappa(w)+w$. Our first theorem shows that $\kappa$ defines a rank preserving bijection from the completely join irreducible elements of $\operatorname{FL}(n)$ to the completely meet irreducible elements.

The (join) rank function $\rho$ defined in the previous section has a particularly simple form in $\operatorname{FL}(n)$. Let $\mathscr{S}$ denote the join closure operator; that is, if $A$ is a subset of a lattice then $\mathscr{S}(A)$ is the set of joins of all finite, nonempty subsets of $A$. $\mathscr{P}$ is defined 
dually. A straightforward induction shows that $D_{k}(\mathrm{FL}(X))=(\mathscr{P} \mathscr{S})^{k} \mathscr{P}(X)$ and $D_{k}^{\prime}(\mathrm{FL}(X))=(\mathscr{S} \mathscr{P})^{k} \mathscr{S}(X)$; see [13]. Thus the $D$-rank function $\rho$ and the $D^{\prime}$-rank function $\rho^{\prime}$ are measures of complexity.

THEOREM 2.1. The map $\kappa$ defines a bijection from the completely join irreducible elements to the completely meet irreducible elements. If $w$ is completely join irreducible, then $\rho(w)=\rho^{\prime}(\kappa(w))$.

Proof. Suppose $w$ is completely join irreducible and $w \succ w_{*}$. To see that $\kappa(w)$ exists let $w_{*}=\prod u_{i}$ be in canonical form. There must be an $i$, say $i=1$, with $u_{1} \neq w$. Now suppose $w v=w_{*}$ for some $v$. Then $w v=w_{*}=\Pi u_{i}$ and so by (the dual of) the refinement property of $\S 1$ we must have $u_{1} \geqslant w$ or $u_{1} \geqslant v$. Hence $u_{1} \geqslant v$; that is, $\kappa(w)=u_{1}$.

It is easy to see that $\kappa(w)$ is completely meet irreducible with upper cover $\kappa(w)^{*}=\kappa(w)+w$. Also if we apply the dual procedure to $\kappa(w)$ we retrieve $w$. Thus $\kappa$ is bijective.

For the statement about ranks we need a lemma.

Lemma 2.2. Let $f: \operatorname{FL}(n) \rightarrow L$ be a lower bounded epimorphism. Then for all $a \in L$, $\rho(a)=\rho(\beta(a))$.

Proof. The proof of Theorem 4.2 of [13] shows that $\beta(a) \in D_{k}(\mathrm{FL}(X))$ implies $a \in D_{k}(L)$. A straightforward induction proves the converse.

Now suppose $w$ is completely join irreducible and $w \succ w_{*}$, and let $\rho(w)=k$. Let $f: \operatorname{FL}(n) \rightarrow L \cong \operatorname{FL}(n) / \psi_{w w_{*}}$ be the canonical map, and recall that $f$ is bounded. (Our proof of this in $\S 4$ will not use any result from this section.) Since $\beta f(w)=w$, by Lemma 2.2 we have $\rho(f(w))=k$. Now $L$, being bounded, is semidistributive, so Theorem 5 of [5] applies to yield $\rho^{\prime}\left(\kappa_{L}(f(w))\right)=k$. We may then use the dual of Lemma 2.2 to obtain $\rho^{\prime}\left(\alpha\left(\kappa_{L}(f(w))\right)\right)=k$. Now it follows easily from the definitions that

$$
\kappa(w)=\alpha\left(\kappa_{L}(f(w))\right) .
$$

Thus $\rho^{\prime}(\kappa(w))=k$, as desired.

The above formula for $\kappa(w)$ will be used below.

Our next theorem shows that to decide if $w$ has a lower cover it suffices to consider join irreducibles.

THEOREM 2.3. Let $w=\sum_{i=1}^{k} w_{i}$ canonically in $\operatorname{FL}(n)$. Then $w$ has a lower cover if and only if some $w_{i} \in \mathrm{CJ}(w)$ has a lower cover.

Proof. Suppose $w \succ u$. Then some element of $\mathrm{CJ}(w)$, say $w_{1}$, is not below $u$. We claim that $w_{1} \succ w_{1} u$. For if $w_{1} u<v \leqslant w_{1}$, then $v \nless u$, so $u+v=w$. From this it follows that for each $w_{i} \in \mathrm{CJ}(w)$, either $w_{i} \leqslant u$ or $w_{i} \leqslant v$. As $w_{1} \nless u$, we have $w_{1} \leqslant v$, whence $w_{1}=v$. Thus $w_{1} \succ w_{1} u$.

Conversely, suppose $w_{1} \in \mathrm{CJ}(w)$ has a lower cover $w_{1 *}$, which will be unique as $w_{1} \in J(\mathrm{FL}(n))$. We claim that $w \succ w \kappa\left(w_{1}\right)$. Note first that for $i>1, w_{i} \leqslant \kappa\left(w_{1}\right)$. For if $w_{i_{0}} \nless \kappa\left(w_{1}\right)$ for some $i_{0}>1$, then by the definition of $\kappa\left(w_{1}\right)$ we would have 
$w_{1} \leqslant w_{1 *}+w_{i_{0}}$, implying $w=w_{1 *}+\sum_{i=2}^{k} w_{i}$, contradicting the refinement property at the end of $\S 1$. Now let $w \kappa\left(w_{1}\right) \leqslant u<w$. Then $u \geqslant w_{1 *}$, but $u \ngtr w_{1}$ because $u \geqslant w \kappa\left(w_{1}\right) \geqslant w_{i}$ for every $i>1$. Thus $u \leqslant \kappa\left(w_{1}\right)$, whence $u=w \kappa\left(w_{1}\right)$. We conclude that $w>w \kappa\left(w_{1}\right)$.

When we start trying to actually determine what the lower covers of a given element in $\operatorname{FL}(n)$ are, it will be useful to have the following formulation of what we have just proved.

COROLlARY 2.4. Let $w=\sum_{i=1}^{k} w_{i}$ canonically in $\mathrm{FL}(n)$. If $w_{i}$ has a lower cover, then $w \succ w \kappa\left(w_{i}\right)$. If $w_{i}$ does not have a lower cover, then $w$ has no lower cover above $\sum_{j \neq i} w_{j}$.

Theorems 2.1 and 2.3, combined with Whitman's solution of the word problem for free lattices [17] and McKenzie's algorithm for testing whether $u \prec v$ in a free lattice (Theorem 6.2 of [14]), show that the predicate " $w$ has a lower cover in $\operatorname{FL}(n)$ " is recursive. For, given $w$, we need only test whether any $w_{i} \in \mathrm{CJ}(w)$ has a lower cover. If $w_{i} \in(\mathscr{P} \mathscr{S})^{k} \mathscr{P}(X)$, then we can use McKenzie's algorithm to check whether $w_{i} u \prec w_{i}$ for any $u \in(\mathscr{S} \mathscr{P})^{k} \mathscr{S}(X)$. Notice that these arguments show that we can effectively find all the lower covers of $w$. While this process is simple enough in principle, we will develop in $\S 4$ a modification of McKenzie's algorithm which will be considerably easier to use.

We note in passing that Theorem 2.1 tells us something about splitting equations. Let $W(X)$ denote the lattice word algebra on $X$, and for $\sigma \in W(X)$ let $\bar{\sigma}$ denote the evaluation of $\sigma$ in $\operatorname{FL}(X)$. If $L$ is a splitting lattice, then the splitting equation for $L$ can be written in the form $\sigma \leqslant \tau$, where $\bar{\sigma}=w$ and $\bar{\tau}=\kappa(w)$ for some $w \in \mathrm{J}(\operatorname{FL}(X))$ with a lower cover. Theorem 2.1 says that $\rho(\bar{\sigma})=\rho^{\prime}(\bar{\tau})$. (Also, recall that by Day's theorem [3] every nontrivial lattice equation implies a splitting equation.)

3. Bounded lattices. In this chapter we show that associated with each lower bounded epimorphism of $\operatorname{FL}(X)$ onto a finite lattice $L$ is a finite set $J$ of join irredicible elements of $\operatorname{FL}(X)$. This set $J$ satisfies a certain natural closure condition. Conversely, any finite closed set of join irredicible elements of $\operatorname{FL}(X)$ gives rise to a finite lower bounded lattice. We show that these finite closed subsets form a lattice under set union and intersection which is dually isomorphic to the filter of Con(FL $(X)$ ) of congruences corresponding to finite lower bounded lattices. At the end of the section we show that a finite lower bounded lattice is also upper bounded if and only if each element of the associated $J$ has a lower cover.

Our ideas are based on the simple observation that if $f: \operatorname{FL}(X) \rightarrow L$ is a lower bounded epimorphism, then $\beta_{f}$ is a join-preserving embedding of $L$ into $\operatorname{FL}(X)$.

If $F$ is a finitely generated lattice and $A$ is a subset of $F$, let $\mathscr{S}_{0}(A)$ denote $\mathscr{S}(A) \cup\{0\}$, that is, the set of all joins of finite subsets of $A$, including $\sum \varnothing=0$. If $A$ is finite, then $\mathscr{S}_{0}(A)$ is of course a lattice, with the join operation inherited from $F$ and the meet in $\mathscr{S}_{0}(A)$ defined by

$$
a \wedge b=\sum\{c \in A: c \leqslant a b \text { in } F\} .
$$


We will be considering lower bounded epimorphisms $f: F \rightarrow L$, where $L$ is finite and $F$ satisfies the condition

$$
\begin{aligned}
& \text { for each } a \in F \text { there is a finite set } T(a) \subseteq F \text { such that every } \\
& \text { join-cover of a refines to a join-cover } U \subseteq T(a) \text {. }
\end{aligned}
$$

Property (E) holds in all finite lattices (clearly) and all projective lattices [9], and hence in all free lattices. Note that if $F$ satisfies $(E)$, then every nontrivial join-cover of $a \in F$ refines to a minimal nontrivial join-cover $U$ of $a$ with $U \subseteq T(a)$.

Moreover, it is not hard to see that if $F$ satisfies (E) and $a \in D_{k}(F)$, then we can choose $T(a)$ so that $T(a) \subseteq D_{k-1}(F) \cup\{a\}$.

Our fundamental result about lower bounded epimorphisms onto finite lattices can now be stated as follows.

THEOREM 3.1. (1) Let $f: F \rightarrow L$ be a lower bounded epimorphism, where $L$ is a finite lattice and $F$ is finitely generated. Let $J=\{\beta(p): p \in J(L)\}$. Note $J \subseteq J(F)$. Then $L \cong \mathscr{S}_{0}(J)$, and $J$ satisfies the closure condition

for each $a \in J$, every join-cover of a refines to a join-cover contained in $J$.

(2) Conversely, let $F$ be finitely generated. If $J$ is a finite subset of $J(F)$ satisfying $(\mathrm{CL})$, then there is a lower bounded epimorphism $f: F \rightarrow \mathscr{S}_{0}(J)$ with $\beta f(w)=w$ for all $w \in J$, given by

$$
f(u)=\sum\{v \in J: v \leqslant u\}
$$

for each $u \in F$.

Proof. (1) Since $\beta$ preserves joins it is easy to see that $\beta$ is an isomorphism from $L$ onto $\mathscr{S}_{0}(J)$.

To see that (CL) holds, let $a \in J$ (so $a=\beta f(a)$ ) and let $U$ be a join-cover of $a$ in $F$. If $a \leqslant u$ for some $u \in U$, this refines to $\{a\} \subseteq J$, so w.l.o.g. the cover is nontrivial. Then $a \leqslant \sum U$ implies $f(a) \leqslant \sum f(U)$, and since $L$ is finite the cover $f(U)$ of $f(a)$ refines to a cover $V \subseteq J(L)$. Then $\beta(V)=\{\beta(v): v \in V\} \subseteq J$ and $a=$ $\beta f(a) \leqslant \sum \beta(V)$, while $V \ll f(U)$ implies $\beta(V) \ll \beta f(U) \ll U$. Thus $J$ satisfies $(\mathrm{CL})$.

(2) Now let $F$ be finitely generated and let $J$ be a finite subset of $J(F)$ satisfying (CL). Then $\mathscr{S}_{0}(J)$ is a lattice, with operations which we will denote by $\wedge$ and $\vee$, and $J$ is the set of join irreducible elements in $\mathscr{S}_{0}(J)$.

Define a $\operatorname{map} f: F \rightarrow \mathscr{S}_{0}(J)$ by

$$
f(u)=\sum\{v \in J: v \leqslant u\} .
$$

This map will occur repeatedly; we call it the standard epimorphism. It is clear that each element of $\mathscr{S}_{0}(J)$ is the least preimage of itself under $f$, so that $f$ is lower bounded. We need to verify that $f$ is in fact a homomorphism. Observe that $f$ is order-preserving, and that $f(u) \leqslant u$ for all $u \in F$. Hence for $u \in F$ and $w \in J$, $w \leqslant u$ if and only if $w \leqslant f(u)$. 
To see that $f$ preserves meets, we calculate (using the above observation)

$$
\begin{aligned}
f(u) \wedge f^{\prime}(v) & =\sum\{w \in J: w \leqslant f(u) f(v)\} \\
& =\sum\{w \in J: w \leqslant u v\}=f(u v) .
\end{aligned}
$$

For joins, we have immediately $f(u+v) \geqslant f(u)+f(v)$. To get the reverse inclusion, it suffices to show that if $w \in J$ and $w \leqslant u+v$, then $w \leqslant f(u)+f(v)$. If $w \leqslant u$ or $w \leqslant v$, this is clear. In the remaining case, $\{u, v\}$ is a nontrivial join-cover of $w$. Since $J$ satisfies (CL), this refines to a minimal nontrivial join-cover $T \subseteq J$. For each $t \in J$ we have $t=f(t)$ by the definition of $f$. Thus $w \leqslant \sum T=\sum_{t \in T} f(t) \leqslant f(u)+$ $f(v)$, as desired.

In free lattices there is a simple syntactic description of subsets of $J(\operatorname{FL}(X))$ satisfying (CL). If $w \in J(\operatorname{FL}(X))$, then $w$ is either a generator or meet reducible, so the canonical form of $w$ is $w=\Pi_{i}\left(\sum_{j} w_{i j}\right) \Pi_{k} x_{k}$, where each $w_{i j} \in J(\operatorname{FL}(X))$ and each $x_{k} \in X$. (Empty meets are simply omitted.) For each $w \in J(\operatorname{FL}(X))$, define $J(w) \subseteq J(\mathrm{FL}(X))$ as follows:

$$
J(w)= \begin{cases}\{w\} & \text { if } w \in \mathscr{P}(X), \\ \{w\} \cup \cup_{i} \cup_{j} J\left(w_{i j}\right) & \text { if } w=\Pi_{i}\left(\sum_{j} w_{i j}\right) \Pi_{k} x_{k} \text { canonically. }\end{cases}
$$

LEMMA 3.2. Let $w \in J(\operatorname{FL}(X))$ with $w=\prod_{i}\left(\sum_{j} w_{i j}\right) \prod_{k} x_{k}$ canonically.

(1) For each $i,\left\{w_{i j}: j=1, \ldots, n_{i}\right\}$ is a minimal nontrivial join-cover of $w$.

(2) If $U$ is a nontrivial join-cover of $w$, then $U$ can be refined to a join-cover of $w$ lying in $J(w)$.

Proof. The proof of (1) is a straightforward application of Whitman's condition (W); cf. [9]. We give a sketch. Canonical form clearly implies $\left\{w_{i j}: j=1, \ldots, n_{i}\right\}$ is a nontrivial cover of $w$. To see that it is minimal suppose $U$ refines $\left\{w_{i j}\right\}$ and $w \leqslant \sum U$. Then $U$ is a nontrival join-cover and so by (W) there is a $t$ with $\sum_{j} w_{t j} \leqslant \sum U$ (since $x_{k}$ is join prime). But $\sum U \leqslant \sum_{j} w_{i j}$. Hence $t=i$ and $\sum U=\sum_{j} w_{i j}=w_{i}$. Since $\left\{w_{i j}\right\}$ is the set of canonical joinands of $w_{i}$ we have $\left\{w_{i j}\right\} \ll U$.

To prove (2), let $U$ be a nontrivial join-cover of $w$. Then by (W) there is an $i$ such that $w_{i j} \leqslant \sum U$ for all $j$. If the latter is a nontrivial covering, then by induction there is a $V_{j} \subseteq J\left(w_{i j}\right)$ with $V_{j} \ll U$ and $\sum V_{j} \geqslant w_{i j}$. If $w_{i j} \leqslant \sum U$ is trivial, then let $V_{j}=\left\{w_{i j}\right\}$. Let $V=\bigcup V_{j}$. Then $V \ll U, V \subseteq J(w)$ and $w \leqslant \Sigma V$, completing the proof.

In particular, Lemma 3.2(2) shows that $\mathrm{FL}(X)$ satisfies the condition (E).

COROllary 3.3. (1) In a free lattice $\mathrm{FL}(X)$, condition (CL) is equivalent to

$$
\text { if } w \in J, \text { then } J(w) \subseteq J .
$$

(2) If $w \in J(\mathrm{FL}(X))$, then $J(w)$ is the smallest subset of $J(\mathrm{FL}(X))$ containing $w$ and satisfying $(\mathrm{CL})$.

Most important for our purposes is then the following

COROllaRy 3.4. A finite lattice $L$ is lower bounded if and only if $L \cong \mathscr{S}_{0}(J)$ for some finite set $J \subseteq J(\mathrm{FL}(X))$ satisfying $(\mathrm{C})$. 


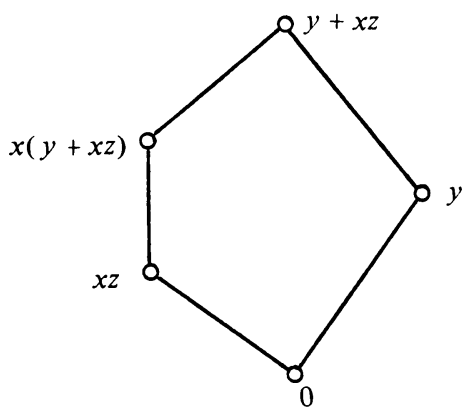

Figure 1

As an example, let $J=\{y, x z, x(y+x z)\} \subseteq J(\mathrm{FL}(3))$. Then $J$ satisfies (C), and $\mathscr{S}_{0}(J)=\{0, y, x z, x(y+x z), y+x z\}$ is isomorphic to $N_{5}$, the five element nonmodular lattice (see Figure 1).

THEOREM 3.5. Let $J$ be a finite subset of $J(\mathrm{FL}(X))$ satisfying the condition $(\mathrm{C})$. If $J^{\prime} \subseteq J$ also satisfies $(\mathrm{C})$, then there is a homomorphism $g: \mathscr{S}_{0}(J) \rightarrow \mathscr{S}_{0}\left(J^{\prime}\right)$. Moreover, every homomorphic image of $\mathscr{S}_{0}(J)$ may be obtained in this way.

Proof. Let $J$ and $J^{\prime}$ be as given above. Then there are lower bounded epimorphisms $f: \operatorname{FL}(\dot{X}) \rightarrow \mathscr{S}_{0}(J)$ and $f^{\prime}: \operatorname{FL}(X) \rightarrow \mathscr{S}_{0}\left(J^{\prime}\right)$, defined as in the proof of Theorem 3.1. We need to show that $\operatorname{ker} f \subseteq \operatorname{ker} f^{\prime}$.

Let $u, v \in \operatorname{FL}(X)$ with $f(u)=f(v)$. Because $f(u)=\sum\{w \in J: w \leqslant u\} \leqslant u$, for every $w \in J$ we have $w \leqslant u$ if and only if $w \leqslant f(u)$. Hence $f(u)=f(v)$ implies $w \leqslant u$ if and only if $w \leqslant v$. As $J^{\prime} \subseteq J$, this means

$$
f^{\prime}(u)=\sum\left\{w \in J^{\prime}: w \leqslant u\right\}=\sum\left\{w \in J^{\prime}: w \leqslant v\right\}=f^{\prime}(v) .
$$

Thus ker $f \subseteq \operatorname{ker} f^{\prime}$.

Now let $g: L \rightarrow L / \theta$ be an epimorphism. Because every homomorphism between finite lattices is bounded, the map $g f: \operatorname{FL}(X) \rightarrow L / \theta$ is lower bounded. Moreover, since the least preimage of a join irreducible element must be join irreducible, we have

$$
J^{\prime}=\left\{\beta_{g f}(p): p \in J(L / \theta)\right\} \subseteq\left\{\beta_{f}(q): q \in J(L)\right\}=J
$$

By Theorem 3.1(1), $J^{\prime}$ satisfies (C) and $L / \theta \cong \mathscr{S}_{0}\left(J^{\prime}\right)$.

Note that a homomorphic image of a finite lower bounded lattice is lower bounded, and a subdirect product of finitely many lower bounded lattices is again lower bounded. Thus $\{\varphi \in \operatorname{Con}(\operatorname{FL}(X)): \operatorname{FL}(X) / \varphi$ is a finite lower bounded lattice $\}$ is a filter in $\operatorname{Con}(\operatorname{FL}(X))$. On the other hand, $\{J \subseteq J(\operatorname{FL}(X)): J$ is finite and satisfies $(C)\}$ is closed under finite unions and intersections. Theorem 3.5 shows that there is a dual isomorphism between these two lattices. Thus we obtain the following

Corollary 3.6. Let $X$ be finite. Then $\{\varphi \in \operatorname{Con}(\operatorname{FL}(X))$ : $\operatorname{FL}(X) / \varphi$ is a finite lower bounded lattice $\}$ is a filter of $\operatorname{Con}(\operatorname{FL}(X))$ which is dually isomorphic to the lattice $\{J \subseteq J(\mathrm{FL}(X))$ : $J$ is finite and satisfies $(\mathrm{C})\}$ under union and intersection. 
In particular, if $J_{i}(1 \leqslant i \leqslant n)$ are finite subsets of $J(\mathrm{FL}(X))$ satisfying $(\mathrm{C})$, then $\mathscr{S}_{0}\left(\cup_{i=1}^{n} J_{i}\right)$ is a subdirect product of the lattices $\mathscr{S}_{0}\left(J_{i}\right)$. Conversely, if $\mathscr{S}_{0}(J)$ is a subdirect product of the lattices $L_{i}(1 \leqslant i \leqslant n)$, then there exist $J_{i} \subseteq J(\operatorname{FL}(X))$ satisfying $(\mathrm{C})$ such that $L_{i} \cong \mathscr{S}_{0}\left(J_{i}\right)$ for $1 \leqslant i \leqslant n$ and $\bigcup_{i=1}^{n} J_{i}=J$.

Before proving the main result of this section, which will connect the representation of finite lower bounded lattices given by Theorem 3.1 and lower covers of join irreducible elements in a free lattice, we need to recall a couple of facts.

LEMMA 3.7. (1) If $L$ is a lower bounded lattice, then $L$ satisfies $\left(\mathrm{SD}_{v}\right)$.

(2) If a finite lower bounded lattice $L$ satisfies $\left(\mathrm{SD}_{\wedge}\right)$, then $L$ is also upper bounded.

Proof. Part (1) is easy and well known. If $L$ is lower bounded and $f: \operatorname{FL}(n) \rightarrow L$, then $u=a+b=a+c$ in $L$ implies $\beta(u)=\beta(a)+\beta(b)=\beta(a)+\beta(c)=\beta(a)$ $+\beta(b) \beta(c)$ since $\operatorname{FL}(n)$ satisfies $\left(\mathrm{SD}_{\vee}\right)$. Thus

$$
u=f \beta(u)=f(\beta(a)+\beta(b) \beta(c))=a+b c .
$$

Part (2) is due to Day and has several proofs, none of which is short enough to be sketched here. (See $[4,5,13,15]$.)

THEOREM 3.8. Let $J$ be a finite subset of $J(\mathrm{FL}(X)$ ) (with $X$ finite) satisfying the condition $(\mathrm{C})$. Then the following are equivalent.

(i) $\mathscr{S}_{0}(J)$ is bounded.

(ii) $\mathscr{S}_{0}(J)$ satisfies $\left(\mathrm{SD}_{\wedge}\right)$.

(iii) Every $w \in J$ has a lower cover in $\operatorname{FL}(X)$.

Proof. The lattice $\mathscr{S}_{0}(J)$ (with $J$ as above) is always lower bounded, so Lemma 3.7 says that $\mathscr{S}_{0}(J)$ is upper bounded if and only if $\mathscr{S}_{0}(J)$ satisfies $\left(\mathrm{SD}_{\wedge}\right)$. Thus (i) is equivalent to (ii).

Next, let us show that (i) implies (iii). Let $f: \operatorname{FL}(X) \rightarrow \mathscr{S}_{0}(J)$ be the standard lower bounded epimorphism, defined as in the proof of Theorem 3.1. By assumption, $f$ is also upper bounded. If $w \in J$, then $w$ is join irreducible in the finite lattice $\mathscr{S}_{0}(J)$, so $w$ has a unique lower cover $w_{\dagger}$ in $\mathscr{S}_{0}(J)$. Since $w=\beta f(w)$, if $v<w$ in $\operatorname{FL}(X)$ then $f(v) \leqslant w_{\dagger}$, whence $v \leqslant \alpha\left(w_{\dagger}\right)$. Thus $w>w \cdot \alpha\left(w_{\dagger}\right)$.

It remains to show that (iii) implies (ii). Assume that every element of $J$ has a lower cover inn $\operatorname{FL}(X)$. By an observation made in $\S 1$, it will suffice to show that for each $w \in J$ there is a unique element $\kappa_{\mathscr{S}_{0}(J)}(w) \in \mathscr{S}_{0}(J)$ which is maximal with respect to being above $w_{\dagger}$ but not above $w$. So let $w \in J$, and let $K=\left\{s \in \mathscr{S}_{0}(J)\right.$ : $s \geqslant w_{\dagger}$ and $\left.s \ngtr w\right\}$. We need to show that $a, b \in K$ implies $a+b \in K$. From this it will follow that $\sum K \in K$, so that $\kappa_{\mathscr{S}_{0}(J)}(w)=\sum K$.

Again, let $f: \operatorname{FL}(X) \rightarrow \mathscr{S}_{0}(J)$ be the standard epimorphism, and let $a, b \in K$. Note that since $a, b \in \mathscr{S}_{0}(J)$ and $\beta(w)=w$, we have $f(a)=a, f(b)=b$, and $f\left(w_{*}\right)=w_{+}$. Therefore $f\left(w_{*}+a\right)=a$ and $f\left(w_{*}+b\right)=b$, so that $w w_{*}+a$ and $w \nless w_{*}+b$ in $\operatorname{FL}(X)$. Thus $w_{*}=w\left(w_{*}+a\right)=w\left(w_{*}+b\right)$, whence we may use $\left(\mathrm{SD}_{\wedge}\right)$ in $\mathrm{FL}(X)$ to obtain $w_{*}=w\left(w_{*}+a+b\right)$, i.e., $w \nless w_{*}+a+b$. In particular, $w \nless a+b$, so $a+b \in K$, as desired. 


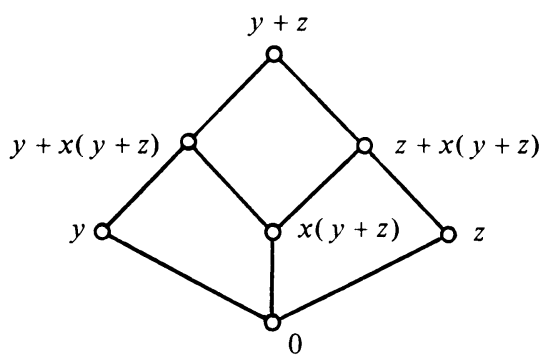

FIGURE 2

As an example of how we can use Theorem 3.8, let $J=\{y, z, x(y+z)\}$. Then $\mathscr{S}_{0}(J)$, which is drawn in Figure 2, fails $\left(\mathrm{SD}_{\wedge}\right)$. Since we know that $y$ and $z$ do have lower covers in $\mathrm{FL}(3)$, we conclude that $x(y+z)$ does not. The nonexistence of a lower cover of $x(y+z)$ in $\mathrm{FL}(3)$ is an unpublished result of R. A. Dean. (See [8] for Dean's proof.)

4. The lattice $L(w)$. Let $w$ be a join irreducible element of $\operatorname{FL}(X)$. We define $L(w)$ to be the lattice $\mathscr{S}_{0}(J(w))$. In this section we show that $w$ has a lower cover in $\operatorname{FL}(X)$ if and only if $L(w)$ satisfies $\left(\mathrm{SD}_{\wedge}\right)$. In fact, if $w$ has a lower cover, then $L(w)$ is isomorphic to the splitting lattice $\operatorname{FL}(X) / \psi_{x^{*} w_{*}}$. We use this to give a syntactic criterion for $w$ to have a lower cover. Several examples are given.

Notice that although $J(w)$ and $L(w)$ are subsets of $\operatorname{FL}(X)$ they essentially are independent of $X$, as long as $w \in \operatorname{FL}(X)$. The one difficulty arises with the element $0=\Pi X$. Technically, $J(\Pi X)$ is not defined since $\Pi X=0$ is not join-irreducible in $\operatorname{FL}(X)$ by our special convention. (Also note that $\Pi X$ is never in $J(w)$.) These difficulties arise since $\Pi X$ has no lower cover in $\operatorname{FL}(X)$ but does have a lower cover in $\mathrm{FL}(Y)$ if $X \subset Y$ (cf. Corollary 4.2 below). This situation could have been avoided by working in the variety of $(0,1)$-lattices, but this is not conventional for the study of free lattices.

The lattices $L(x(y+x z))$ and $L(x(y+z))$ were used as examples in the previous chapter (see Figures 1 and 2). We will give more examples of the lattices $L(w)$ with some applications later in this section.

THEOREM 4.1. Let $w \in J(\operatorname{FL}(X))$ with $X$ finite. Let $w_{\dagger}$ denote the lower cover of $w$ in $L(w)$. Then:

(1) $L(w)$ is a finite, lower bounded, subdirectly irreducible lattice with $w / w_{\dagger}$ as a critical prime quotient.

(2) $w$ has a lower cover in $\operatorname{FL}(X)$ if and only if $L(w)$ satisfies $\left(\mathrm{SD}_{\wedge}\right)$.

(3) If $K$ is any lattice for which there exists an epimorphism g: $\operatorname{FL}(X) \rightarrow K$ such that $g(u)<g(w)$ whenever $u<w$, then $L(w)$ is a homomorphic image of $K$ (i.e., $\operatorname{ker} f$ is the unique largest congruence on $\mathrm{FL}(X)$ with the property that $(u, w) \notin \theta$ whenever $u<w$, where $f: \operatorname{FL}(X) \rightarrow L(w)$ is the standard epimorphism).

Proof. (1) $L(w)$ is lower bounded because $J(w)$ satisfies (C). If $\theta$ is a nontrivial congruence relation on $L(w)$, then by Theorem 3.5, $\theta$ is the kernel of a homomorphism $g: L(w) \rightarrow \mathscr{S}_{0}\left(J^{\prime}\right)$ for some $J^{\prime}$ properly contained in $J(w)$ which satisfies (C). 
The map $g$ sends each $u \in L(w)$ to $g(u)=\sum\left\{v \in J^{\prime}: v \leqslant u\right\}$. Since $J^{\prime} \varsubsetneqq J(w)$, we must have $w \notin J^{\prime}$, whence

$$
g(w)=\sum\left\{v \in J^{\prime}: v \leqslant w\right\}=\sum\left\{v \in J^{\prime}: v<w\right\}=g\left(w_{\dagger}\right) .
$$

Thus $L(w)$ is subdirectly irreducible with $w / w_{\dagger}$ as a critical prime quotient.

(2) If $L(w)$ satisfies $\mathrm{SD}_{\wedge}$, then by Theorem 3.8, $w$ has a lower cover in $\operatorname{FL}(X)$.

Conversely, assume $w$ has a lower cover $w_{*}$ in $\operatorname{FL}(X)$. Then it follows from (1) that $L(w) \cong \mathrm{FL}(X) / \psi_{w w_{*}}$. On the other hand, since $w$ is an upper cover of $w_{*}$, the dual of Theorem 2.3 says that some $u$ in the canonical meet representation of $w_{*}$ has an upper cover $u^{*}$. In fact, choosing $u$ as in the dual of the proof of Theorem 2.3, $u^{*} / u$ and $w / w_{*}$ are projective prime quotients in $\operatorname{FL}(X)$, so that in particular $\psi_{u^{*} u}=\psi_{w w_{*}}$. Now $u \in M(\operatorname{FL}(X))$; if we define $M(u) \subseteq M(\operatorname{FL}(X))$ dually to $J(w)$ and $L^{\prime}(u)=\mathscr{P}_{1}(M(u))$ dually to $L(w)$, then $L^{\prime}(u)$ is a finite, subdirectly irreducible, upper bounded lattice. Moreover, since $u$ has upper cover,

$$
L^{\prime}(u) \cong \operatorname{FL}(X) / \psi_{u^{*} u}=\operatorname{FL}(X) / \psi_{w w_{*}} \cong L(w)
$$

By the dual of Lemma 3.7, $L^{\prime}(u)$ satisfies $\mathrm{SD}_{\wedge}$, and hence so does $L(w)$.

REMARK. Parts (1) and (2) of Theorem 4.1, when combined with Lemma 3.7, give a new proof of McKenzie's result [14] that $\operatorname{FL}(X) / \psi_{w w_{*}}$ is a finite, subdirectly irreducible, bounded lattice. The element $u$ found in the above proof of (2) is, of course, $\kappa(w)$.

(3) If $w$ has a lower cover, then this is an immediate consequence of $L(w) \cong$ $\operatorname{FL}(X) / \psi_{w w_{*}}$. We are seeking the analogous statement for the case when $w$ has no lower cover in $\operatorname{FL}(X)$.

Let $F, K$ be lattices and let $g: F \rightarrow K$ be an epimorphism. Then $g$ extends to an epimorphism of the ideal lattices, $\bar{g}: \mathscr{I}(F) \rightarrow \mathscr{I}(K)$, given by $\bar{g}(I)=\{g(i): i \in I\}$. (This is straightforward to verify; see also [7 and 13].) If $w \in J(F)$, then in $\mathscr{I}(F)$ the principal ideal $w / 0$ covers the ideal $U=\{u \in F: u<w\}$. Hence in $\operatorname{Con}(\mathscr{I}(F))$ there is a unique maximal congruence $\bar{\psi}_{w}$ with the property that $(w, U) \notin \bar{\psi}_{w}$. Moreover, if $g: F \rightarrow K$ is such that $g(u)<g(w)$ for all $u<w$, then $\operatorname{ker} \bar{g} \leqslant \bar{\psi}_{w}$, whence in $\operatorname{Con}(F)$ we have $\operatorname{ker} g=\left.\operatorname{ker} \bar{g}\right|_{F} \leqslant\left.\bar{\psi}_{w}\right|_{F}$. In other words, $\left.\bar{\psi}_{w}\right|_{F}$ is the unique maximal congruence $\theta$ on $F$ such that $(u, w) \notin \theta$ for all $u<w$.

Now let $w \in J(\operatorname{FL}(X))$ and consider the standard lower bounded epimorphism $f$ : $\operatorname{FL}(X) \rightarrow L(w)$. Since $\beta f(w)=w$, we have $(u, w) \notin \operatorname{ker} f$ for every $u<w$. Since $L(w)$ is subdirectly irreducible with $w / w_{\dagger}$ as a critical prime quotient, $\operatorname{ker} f$ is maximal in $\operatorname{Con}(\mathrm{FL}(X))$ with respect to this property. By the above remarks, we conclude that $\operatorname{ker} f=\left.\bar{\psi}_{w}\right|_{\mathrm{FL}(X)}$ and $L(w) \cong \mathrm{FL}(X) /\left(\bar{\psi}_{w} \mid \operatorname{FL}(X)\right)$. Thus whenever $g$ : $\operatorname{FL}(X) \rightarrow K$ with $g(u)<g(w)$ for all $u<w$, we have $\operatorname{ker} g \leqslant \operatorname{ker} f$, as desired.

For $w \in \operatorname{FL}(X)$, let $\operatorname{var}(w)$ denote the set of variables involved in the canonical representation of $w$, i.e., $\operatorname{var}(w)$ is the smallest subset $S$ of $X$ such that $w$ is in the sublattice generated by $S$.

Corollary 4.2. Let $w \in J(\mathrm{FL}(X))$ with $X$ finite.

(1) If some $u \in J(w)$ fails to have a lower cover in $\operatorname{FL}(X)$, then so does $w$. 


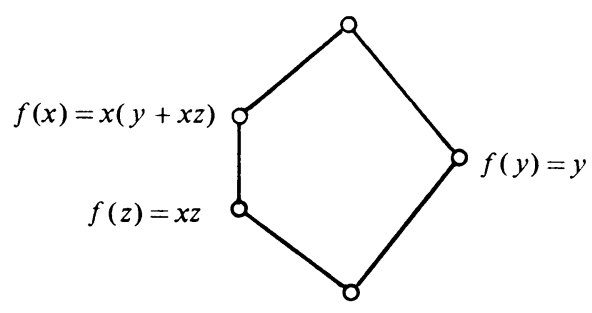

FIGURE 3

(2) If $w$ has a lower cover in $\operatorname{FL}(X)$ and $X \subseteq Y$ with $Y$ finite, then $w$ has a lower cover in $\operatorname{FL}(Y)$. Conversely, if $w$ has a lower cover in $\operatorname{FL}(X)$ and $\operatorname{var}(w) \subseteq Z \subseteq X$, then unless $w=\Pi Z, w$ has a lower cover in $\operatorname{FL}(Z)$.

Proof. (1) By Theorem 3.5, if $u \in J(w)$, then $L(u)$ is a homomorphic image of $L(w)$. Now every homomorphic image of a finite bounded lattice is bounded. Thus if $L(w)$ is bounded, so is $L(u)$ for every $u \in J(w)$. Hence $w$ can have a lower cover in $\operatorname{FL}(X)$ only if every $u \in J(w)$ does.

(2) This follows immediately from Theorem 4.1(2) and the observation that, if $w$ is not a meet of generators, then $L(w)$ depends only on the elements in the sublattice of $\operatorname{FL}(X)$ generated by $\operatorname{var}(w)$. For $\varnothing \neq Z \subset X$, we have $J(\Pi Z)=\{\Pi Z\}$, whence $L(\Pi Z) \cong \mathbf{2}$, and as is well known $\Pi Z>\Pi Z \cdot \Sigma(X \backslash Z)$ in $\operatorname{FL}(X)$. However, as remarked above, $L(\Pi X)$ is not defined since $\Pi X$, the zero element of $\operatorname{FL}(X)$, is not in $J(\mathrm{FL}(X))$, and of course $\Pi X$ has no lower cover in $\operatorname{FL}(X)$.

Note that while the existence of a lower cover of $w$ in $\operatorname{FL}(X)$ in general depends only on the set $\operatorname{var}(w)$, the element $w_{*}$ actually covered by $w$ does depend on the set $X$. Recall $w_{*}=w \kappa(w)$ and $\kappa(w)=\alpha_{f} \kappa_{L(w)}(w)$, where $f: \operatorname{FL}(X) \rightarrow L(w)$ is the standard lower bounded epimorphism. For $t \in X \backslash \operatorname{var}(w)$, note $f(t)=0$. Using the construction for $\alpha_{f}$, it is not hard to see that if $\kappa(w)=p\left(x_{1}, \ldots, x_{n}\right)$ in $\operatorname{FL}(\operatorname{var}(w))$, then in $\mathrm{FL}(X)$ the new $\kappa(w)$ is given (not necessarily in canonical form) by $p\left(x_{1}+s, \ldots, x_{n}+s\right)$, where $s=\Sigma(X \backslash \operatorname{var}(w))$.

Our results make it a relatively easy task to determine the lower and upper covers of a given element in $\mathrm{FL}(X)$. As appropriate, we can use Theorem 2.3, Theorem 4.1(2), or Corollary 4.2(1), and of course their duals. Recall also that if $w \in J(\operatorname{FL}(X))$ has a lower cover, then $w_{*}=w \kappa(w)$, while $\kappa(w)$ is not defined if $w_{*}$ does not exist. In Table 1, we give $\kappa(w)$ and the upper and lower covers for some join irreducible elements in FL(3). We will give below proofs for some of the entries, and leave the proofs of others to the reader.

For example, if $w=x(y+z)$, then $J(w)=\{x(y+z), y, z\}$. Thus $L(x(y+z))$ is the lattice drawn in Figure 2. This lattice fails $\left(\mathrm{SD}_{\wedge}\right)$, so as we concluded in $\S 3$, $x(y+z)$ does not have a lower cover. By the dual of Theorem 2.3, the upper covers of $w$ are of the form $w+\kappa^{\prime}\left(w_{i}\right)$ for each $w_{i}$ in the canonical meet representation of $w$ which has an upper cover. In this case $w_{1}=x$ and $\kappa^{\prime}(x)=y z$, yielding the upper cover $x(y+z)+y z$; and $w_{2}=y+z$ with $\kappa^{\prime}(y+z)=x$, yielding the upper cover $x(y+z)+x=x$. 


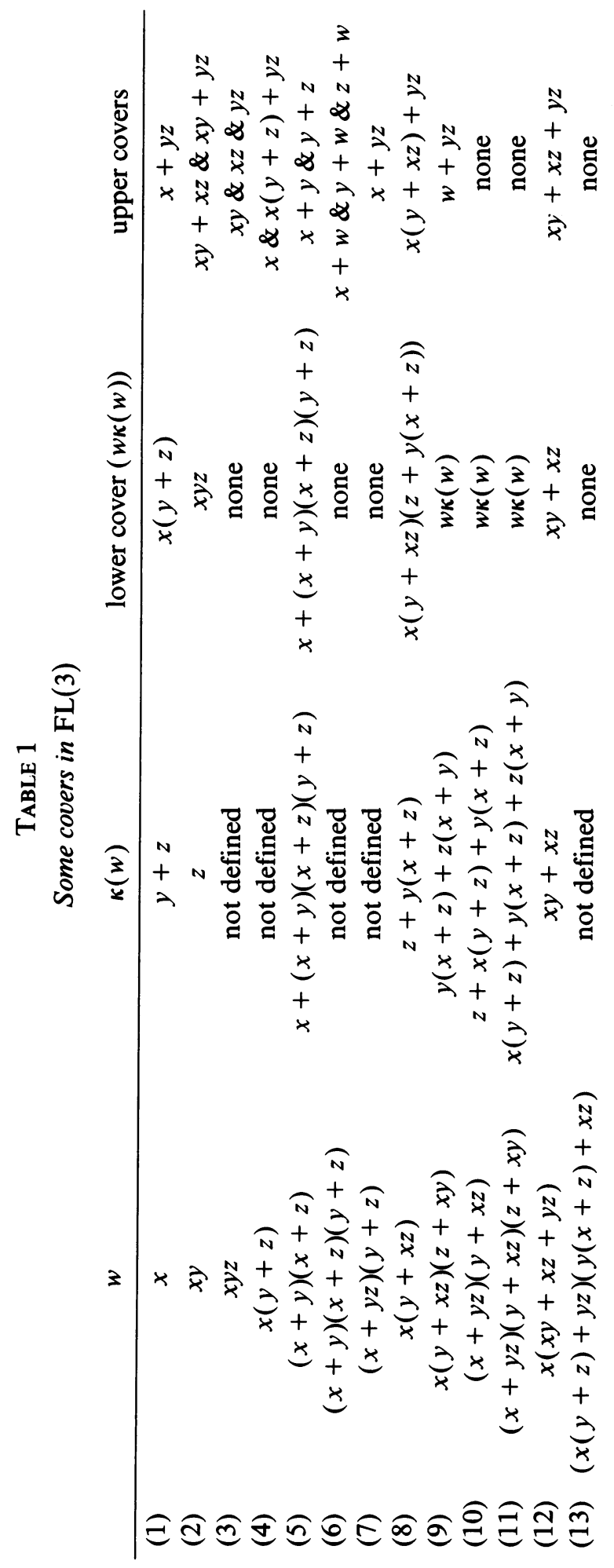


If $w=x(y+x z)$, then $J(w)=\{x(y+x z), y, x z\}$. The lattice $L(x(y+x z))$, which is drawn in Figure 1 above, is isomorphic to the five-element nonmodular lattice $N$. This lattice of course satisfies $\left(\mathrm{SD}_{\wedge}\right)$, so we conclude that $x(y+x z)$ has a lower cover in $\operatorname{FL}(3)$. To find $\kappa(x(y+x z))$, we first construct the standard epimorphism $f: \operatorname{FL}(3) \rightarrow L(x(y+x z))$. By definition $f(u)=\sum\{v \in J(x(y+x z))$ : $v \leqslant u\}$, so we have Figure 3. Recall that $\kappa(w)=\alpha_{f} \kappa_{L\left(w^{\prime}\right)}(w)$. In this case $\kappa_{L\left(w^{\prime}\right)}(w)=$ $x z$, while $\alpha(u)$ is always found by applying the dual of the algorithm given in $\S 1$ for finding $\beta(u)$. Doing this yields $\kappa(x(y+x z))=z+y(x+z)$.

As in the preceding case, we obtain an upper cover of $x(y+x z)$ in $x(y+x z)+$ $\kappa^{\prime}(x)=x(y+x z)+y z$. However, $w_{2}=y+x z$ has no upper cover (by the dual of the first argument), so $x(y+x z)$ has no upper cover below $x$.

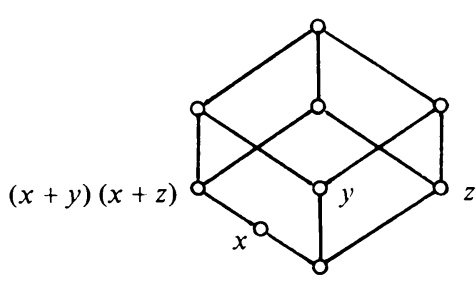

$L((x+y)(x+z)$

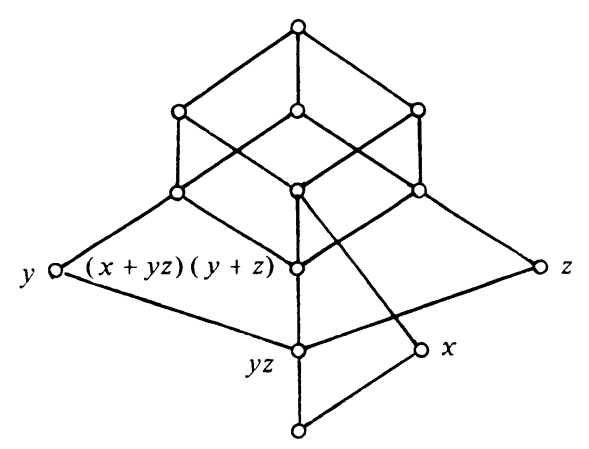

$L((x+y z)(y+z))$

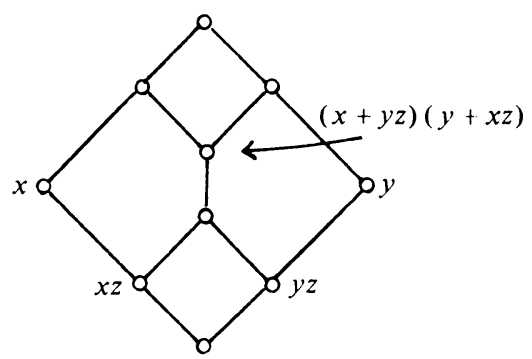

$L((x+y z)(y+x z))$

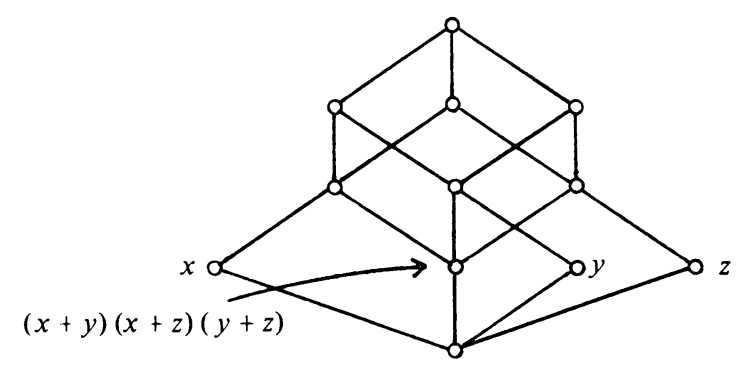

$L((x+y)(x+z)(y+z))$

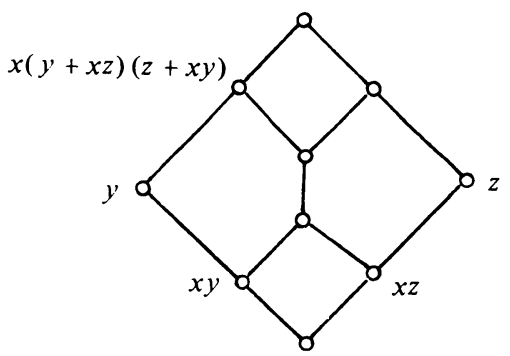

$L(x(y+x z)(z+x y))$

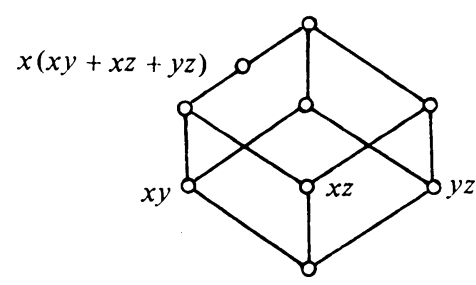

$L(x(x y+x z+y z))$

FIGURE 4 
Figure 4 gives several more examples of lattices $L(w)$ with $w \in J(\operatorname{FL}(3))$. The reader is invited to try extracting from these lattices some of the information given in Table 1.

As a final example, let us show that the element

$$
w=(x(y+z)+y z)(y(x+z)+x z)
$$

has neither an upper nor a lower cover in FL(3). (This answers a question of David Kelly about the existence of such elements.) First note that $x(y+z)$, which has no lower cover, is in $J(w)$. By Corollary 4.2(1), this implies that $w$ has no lower cover. On the other hand, by the dual of (7) in Table $1, x(y+z)+y z$ has no upper cover, and symmetrically the same is true for $y(x+z)+x z$. Since these are the elements in the canonical meet representation of $w$, we conclude that $w$ has no upper cover.

We continue this chapter with syntactic versions of the algorithm for determining whether $w \in J(\operatorname{FL}(X))$ has a lower cover in $\operatorname{FL}(X)$, and for finding $\kappa(w)$ when it exists.

TheOrem 4.3. Let $w \in J(\operatorname{FL}(X))$ with $X$ finite. Let $w_{\dagger}=\sum\{u \in J(w): u<w\}$, and define $K(w)=\left\{v \in J(w): w_{\dagger}+v \ngtr w\right\}$.

(i) every $u \in J(w) \backslash\{w\}$ has a lower cover in $\operatorname{FL}(X)$, and

(ii) $w \nless \sum K(w)$.

The proof is an easy combination of Theorem 4.1(2) and Corollallry 4.2(1), and will be left to the reader. Closely related to Theorem 4.3 is the following useful necessary condition for $w \in J(\mathrm{FL}(X))$ to have a lower cover.

TheOREM 4.4. Let $w \in J(\mathrm{FL}(X))$, where $X$ is finite, with $w=\Pi_{i}\left(\sum_{j} w_{i j}\right) \Pi_{k} x_{k}$ canonically. If $w$ has a lower cover, then for each $i$ there is exactly one $j$ with $w_{i j} \nless w$.

Proof. Let $w_{i}=\sum_{j} w_{i j}$. If $w$ has a lower cover, then $\kappa(w)$ exists, and since $w \leqslant w_{i}$ we have $w_{i} \nless \kappa(w)$. Thus for each $i$ there exists at least one $j$ such that $w_{i j} \nless \kappa(w)$, say w.l.o.g. $w_{i 1} \nless \kappa(w)$ for each $i$.

Fix any index $i_{0}$. Applying Whitman's condition (W) to the inclusion

$$
w=\prod_{i} w_{i} \cdot \prod_{k} x_{k} \leqslant w_{*}+w_{i_{0}}
$$

we easily obtain $w_{i} \leqslant w_{*}+w_{i_{0} 1}$ for some $i$. Since $w_{*}+w_{i_{0} 1} \leqslant w+w_{i_{0}}=w_{i_{0}}$, this implies $w_{i_{0}}=w_{*}+w_{i_{0}}$. However, $w_{i_{0}}=\sum_{j=1}^{m} w_{i_{0} j}$ canonically, which means that $\left\{w_{i_{0} j}: j=1, \ldots, m\right\}$ refines $\left\{w_{*}, w_{i_{0} 1}\right\}$. Thus $w_{i_{0} j} \leqslant w_{*}$ for every $j>1$. Since $i_{0}$ was arbitrary this proves the theorem.

By Lemma 3.2, $\left\{w_{i j}: j=1, \ldots, m\right\}$ is a minimal nontrivial join-cover of $w$ for each $i$. Theorem 4.4 can then be rephrased in the terminology introduced by Jonsson (see $\left[13,15\right.$, or 16]) as follows. Let $w=\Pi_{i}\left(\sum_{j} w_{i j}\right) \Pi_{k} x_{k}$ have a lower cover. Then for each $i, w B w_{i j}$ for exactly one $j$, and $w A w_{i j}$ for the remaining $j$ 's.

The converse of Theorem 4.4 is false. For example

$$
w=(x+y)(x+(y+z)(z+t))
$$

satisfies the condition of Theorem 4.4, but does not have a lower cover. However, for elements of very low complexity the converse does hold. 
THEOREM 4.5. Let $w \in \mathscr{P} \mathscr{S P}(X)$ be a join irreducible element with $w=$ $\Pi_{i}\left(\Sigma_{j} w_{i j}\right) \Pi_{k} x_{k}$ canonically. Then $w$ has a lower cover in $\mathrm{FL}(X)(X$ finite $)$ if and only if for each $i$ there is exactly one $j$ such that $w_{i j} \ldots w$.

Proof. Let $w \in \mathscr{P} \mathscr{S} \mathscr{P}(X)$ satisfy the condition. Then $J(w)=\{w\} \cup\left\{w_{i j}: 1 \leqslant i\right.$ $\left.\leqslant n, 1 \leqslant j \leqslant m_{i}\right\}$, and in applying Theorem 4.3 we find that $w_{i j} \in K(w)$ if and only if $w_{i j}<w$. Hence $w$ has a lower cover.

At this point we pause for a fundamental observation. If $w \in J(\operatorname{FL}(X))$ and $f$ : $\mathrm{FL}(X) \rightarrow L(w)$ is the standard epimorphism, then each canonical meetand of $w$ is either a generator or an element of the form $\sum_{u \in U} \beta(u)$, where $U$ is a minimal nontrivial join-cover of $w$ in $L(w)$ (since $w=\beta_{f}(w)$ ), although not necessarily all such elements are canonical meetands of $w$. If $w$ is completely join irreducible, then since ker $f=\psi_{w^{\prime} w_{*}}=\psi_{\kappa(w)^{*} \kappa(w)}$, it follows by duality that the canonical joinands of $\kappa(w)$ are generators and elements of the fornı $\prod_{v \in V} \alpha(v)$, where $V$ is a maximal nontrivial meet-cover of $\kappa_{L}(w)$ in $L(w)$. On the other hand, if $v$ is a meet irreducible element of $L(w)$ with $w$ completely join irreducible, then $\alpha(v)=\kappa(u)$ for some $u \in J(w)$ (viz., for $u=\kappa_{L(w)}^{\prime}(v)$ ) because there is a one-to-one correspondence between join irreducibles and meet irreducibles in $L(w)$, and each $L(u)(u \in J(w))$ is a homomorphic image of $L(w)$. It follows that the canonical joinands of $\kappa(w)$ are either generators or elements of the form $\Pi_{u \in U} \kappa(u)$, where $U \subseteq J(w)-\{w\}$. This will prove to be a useful observation. For example, we obtain a syntactic algorithm for finding $\kappa(w)$ when it exists.

THEOREM 4.6. Let $w \in J(\operatorname{FL}(X))$, with $X$ finite, have a lower cover. Then $\kappa(w)$ may be found as follows.

(1) Find $\kappa_{L\left(w^{\prime}\right)}=\sum K(w)$ as in Theorem 4.3.

(2) Form $M_{0}=\{\kappa(u): u \in J(w)-\{w\}\}$.

(3) Let $k^{\dagger}=\prod\left\{v \in M_{0}: v \geqslant \kappa_{L\left(w^{\prime}\right)}(w)\right\}$.

(4) Then $\kappa(w)=\sum\left\{x \in X: x+w_{+} \neq w\right\}+\sum\left\{k^{\dagger} v: v \in M_{0}, w \nless v\right\}$.

(5) Put $\kappa(w)$ in canonical form.

Proof. Note that $\kappa_{L\left(w^{\prime}\right)}(w)$, as found in (1), is $\beta f(\kappa(w))$ for the standard epimorphism $f$. Let $g$ : $\mathrm{FL}(X) \rightarrow \mathscr{P}_{1}(M(\kappa(w)))$ be defined dually to the standard epimorphism $f: \operatorname{FL}(X) \rightarrow \mathscr{S}_{0}(J(w))$. Note that $f$ and $g$ have the same kernel, viz., $\psi_{w^{\prime} w_{*}}=\psi_{\kappa\left(w^{\prime}\right)^{*} \kappa(w)}$. Our object is to find $\kappa(w)=\alpha g(\kappa(w))=\alpha f(\kappa(w))$.

By the observation preceding the statement of the theorem, in (2) we are finding $M_{0}=M(\kappa(w))-\{\kappa(w)\}$.

In (3) we are finding $k^{\dagger}=\alpha f\left(\kappa(w)^{*}\right)$. To see this, let us show that for $v \in M_{0}$, $v \geqslant \kappa_{L\left(w^{\prime}\right)}(w)$ if and only if $v \geqslant \kappa(w)^{*}=w+\kappa(w)$. Let $h: \operatorname{FL}(X) \rightarrow \mathscr{P}_{1}\left(M_{0}\right)$ be the standard epimorphism, and observe that $\mathscr{P}_{1}\left(M_{0}\right) \cong \mathscr{P}_{1}(M(\kappa(w))) / \theta$, where $\theta=$ $\operatorname{con}\left(\kappa(w), \alpha g\left(\kappa(w)^{*}\right)\right)$. If $v \in M_{0}$ and $v \geqslant \kappa_{L\left(w^{\prime}\right)}(w)$, then using $\operatorname{ker} h>\operatorname{ker} g=\operatorname{ker} f$ we obtain

$$
v=h(v) \geqslant h\left(\kappa_{L(w)}(w)\right)=h(\kappa(w))=h\left(\alpha g\left(\kappa(w)^{*}\right)\right)=h\left(\kappa(w)^{*}\right) \geqslant \kappa(w)^{*},
$$

as desired. The converse is obvious as $\kappa_{L\left(w^{\prime}\right)}(w)=\beta f(\kappa(w)) \leqslant \kappa(w)^{*}$, so the claim is proved. 
Note that if $\Pi_{j} v_{j}$ is a canonical summand of $\kappa(w)$, then for each $j$ we have $v_{j} \in M_{0}$, and by the dual of Theorem 4.4 there is a unique $j$ with $v_{j} \neq \kappa(w)$, or equivalently, with $v_{j} \neq k^{\dagger}$. Moreover, with this $v_{j}$ in mind we note that for $v \in M_{0}$, $k^{\dagger} v \leqslant \kappa(w)$ if and only if $v \ngtr \kappa^{\prime}(\kappa(w))=w$. Now (4) just gives the usual algorithm for finding $\alpha(g(\kappa(w)))=\kappa(w)$ with the above considerations taken into account.

On the other hand, there is no reason to expect this expression for $\kappa(w)$ to necessarily be in canonical form, and sometimes it is not; hence (5) is included.

As an example, let us find $\kappa((x t+z t)(y t+z t))$ in $\operatorname{FL}(4)$. Now $J(w)=$ $\{w, x t, y t, z t\}$ and $\kappa_{L(w)}(w)=z t$. Moreover, $M_{0}=\{y+z, x+z, x+y\}$; whence $k^{\dagger}=(x+z)(y+z)$. On the other hand $x+y \neq w$, and we separately check that $w_{\dagger}+z=z \ngtr w$. Thus (4) gives us $\kappa(w)=z+(x+z)(y+z)(x+y)$, which is in fact already in canonical form.

The advantage of our syntactic algorithms (Theorems 4.3-4.6) is that they extract the crucial information from $L(w)$ without requiring that the lattice be constructed. In fact, even though the algorithm of Theorem 4.6 looks complicated, we have programmed a microcomputer to do it using muLISP, and the program runs quite quickly. The reader is encouraged to try his hand with the algorithms above on some of the examples in Table 1.

Another class of examples, which we shall use in $\S 10$ is constructed as follows. In $\operatorname{FL}(x, y, z)$ let $y_{0}=y, z_{0}=z$ and

$$
y_{i+1}=y\left(z_{i}+x y\right), \quad z_{i+1}=z\left(y_{i}+x z\right), \quad w_{i}=\left(y_{i}+x z\right)\left(z_{i}+x y\right) .
$$

Then each $y_{n}, z_{n}$ and $w_{n}$ is completely join irreducible by Theorem 4.3. Indeed it is easy to see that $J\left(y_{n}\right)=\left\{x y, x z, y_{n}, z_{n-1}, y_{n-2}, z_{n-3}, \ldots\right\}, y_{n \dagger}=x y$ and $K\left(y_{n}\right)=$ $\{x y, x z\}$. Thus by Theorem $4.3, y_{n}$ is completely join irreducible. Now $J\left(w_{n}\right)=\left\{w_{n}\right\}$ $\cup J\left(y_{n}\right) \cup J\left(z_{n}\right), w_{n \dagger}=x y+x z$ and $K\left(w_{n}\right)=\{x y, x z\}$ and thus $w_{n}$ is completely join irreducible.

We close this section with a theorem on the canonical meetands of $w_{*}$.

THEOREM 4.7. Let $w$ be a completely join irreduciblle element of $\operatorname{FL}(X), X$ finite. Let $w=w_{1} \cdots w_{m}$ canonically. Then the canonical meetands of $w_{*}$ are $\{\kappa(w)\} \cup\left\{w_{i}\right.$ : $\left.w_{i} \ngtr \kappa(w)\right\}$.

Proof. Let $w_{*}=\prod_{j=1}^{t} u_{j}$ canonically. Since $w_{*}=w \kappa(w)$, the refinement property described at the end of $\S 1$ tells us that $\left\{u_{1}, \ldots, u_{t}\right\} \gg,\{w, \kappa(w)\}$. Clearly there must be an $i$, say $i=1$, with $u_{i} \neq w$. Then $u_{1} \geqslant \kappa(w)$ and $u_{1} \neq w$, which implies $u_{1}=\kappa(w)$. Also $w_{*}=w_{1} \cdots w_{m} \kappa(w)$. Hence $\left\{u_{i}: i \geqslant 2\right\} \gg\left\{w_{1}, \ldots, w_{m}\right\}$. Renumber so that $\left\{w_{i}: w_{i} \neq \kappa(w)\right\}=\left\{w_{1}, \ldots, w_{n}\right\}$ and note $\left\{u_{i}: i \geqslant 2\right\} \gg\left\{w_{1}, \ldots, w_{n}\right\}$.

Suppose $\rho(w)=k$, i.e., $w \in D_{k}(\operatorname{FL}(X))$. By the description of $D_{k}(\operatorname{FL}(X))$ and $D_{k}^{\prime}(\mathrm{FL}(X))$ given at the beginning of $\S 2$ we see that $w \in D_{k+1}^{\prime}(\operatorname{FL}(X))$ and that $w_{i} \in D_{k}^{\prime}(\mathrm{FL}(X)), i=1, \ldots, m$. Since $w_{i} \geqslant w \geqslant w_{*}=\prod u_{i},\left\{u_{1}, \ldots, u_{t}\right\}$ is a dual cover of $w_{i}$. If $w_{i} \geqslant \prod u_{i}$ is nontrivial, then there is a $V_{i} \subseteq D_{k-1}^{\prime}(\operatorname{FL}(X))$ with $V_{i} \gg\left\{u_{1}, \ldots, u_{t}\right\}$ and $w_{i} \geqslant \Pi V_{i}$.

Set $V_{i}=\left\{w_{i}\right\}$ if $w_{i} \geqslant \prod u_{j}$ is a trivial cover, and let $V=\bigcup_{i=1}^{m} V_{i}$. Then $V \gg$ $\left\{u_{1}, \ldots, u_{t}\right\}$ and each $a \in V$ is either some $w_{i}$ or in $D_{k-1}^{\prime}(\operatorname{FL}(X))$. Also $w \geqslant \Pi V \geqslant$ $\Pi u_{j}=w_{*}$. If $\Pi V=w_{*}$, then $\left\{u_{1}, \ldots, u_{t}\right\} \gg V \gg\left\{u_{1}, \ldots, u_{t}\right\}$ which implies 
$\left\{u_{1}, \ldots, u_{t}\right\} \subseteq V$. Then $\kappa(w)=u_{1} \in V$ and thus $\kappa(w) \in D_{k-1}^{\prime}(\operatorname{FL}(X))$, contradicting Theorem 2.1. Hence $w=\Pi V$ and thus $\left\{w_{1}, \ldots, w_{m}\right\} \gg V \gg\left\{u_{1}, \ldots, u_{t}\right\}$. Combining this with the above, we easily obtain $\left\{u_{j}: j \geqslant 2\right\} \gg\left\{w_{1}, \ldots, w_{n}\right\} \gg\left\{u_{j}\right.$ : $j \geqslant 2\}$. Since both these sets are antichains, we have $\left\{w_{1}, \ldots, w_{n}\right\}=\left\{u_{j}: j \geqslant 2\right\}$, proving the theorem.

5. Day's theorem revisited. Alan Day proved in [3] that every finitely generated free lattice is weakly atomic. That is to say, if $u>v$ in $\operatorname{FL}(X)$ with $X$ finite, then there exist $s, t \in \mathrm{FL}(X)$ with $u \geqslant s \succ t \geqslant v$. The crucial observation in Day's proof is that "doubling an interval" in a lattice preserves boundedness.

In this section we will present a new proof of Day's result, based on the methods developed in $\$ 3$. In the end, our variation is no simpler than Day's original proof, but it is constructive and does yield more information about the nature of the coverings which can be found in $u / v$.

We will derive Day's theorem from the following results.

THEOREM 5.1. If $u \nless v$ in $\operatorname{FL}(X)$ with $X$ finite, then there exist a finite bounded lattice $L$ and an epimorphism $f: \operatorname{FL}(X) \rightarrow L$ such that $f(u) \nless f(v)$. Moreover, if $\rho^{\prime}(u)=m$ and $\rho(v)=n$ with $m+n \geqslant 1$, then $L$ may be chosen so that $J(L) \subseteq$ $D_{m+n-1}(L)$.

In fact, our proof of Theorem 5.1 gives the following (equivalent) statement.

Corollary 5.2. If $u \nless v$ in $\operatorname{FL}(X)$ with $X$ finite, then there exists $q \in J(\operatorname{FL}(X))$ with a lower cover such that $q \leqslant u$ and $v \leqslant \kappa(q)$. Moreover, if $\rho^{\prime}(u)=m$ and $\rho(v)=n$ with $m+n \geqslant 1$, then $q$ may be chosen so that $\rho(q) \leqslant m+n-1$.

From Corollary 5.2 we immediately obtain

$$
v \leqslant \kappa(q)(q+v) \prec q+v \leqslant u+v
$$

in $\operatorname{FL}(X)$, which for $u>v$ is Day's theorem. Conversely, if $u \geqslant a>b \geqslant v$ in $\operatorname{FL}(X)$, then $q \nless b$ for some $q \in \mathrm{CJ}(a)$. As in the proof of Theorem 2.3, $q \succ b q$. Hence $\kappa(q)$ exists, $q \leqslant a \leqslant u$, and $v \leqslant b \leqslant \kappa(q)$. Thus every cover in $u / v$ arises in this way.

Proof of Theorem 5.1. For each pair $(u, v)$ of elements of $\operatorname{FL}(X)$ with $u \nless v$, we will construct a finite set $J(u, v) \subseteq J(\mathrm{FL}(X))$ with the following properties:

(i) $J(u, v)$ satisfies (C), and every $p \in J(u, v)$ has a lower cover in $\operatorname{FL}(X)$.

(ii) There is a $q \in J(u, v)$ such that $q \leqslant u$ and $v \leqslant \kappa(q)$.

(iii) If $\rho^{\prime}(u)=: n$ and $\rho(v)=n$ with $m+n \geqslant 1$, then $\rho(p) \leqslant m+n-1$ for every $p \in J(u, v)$.

Let $K(u, v)=S_{0}(J(u, v))$. Condition (i) makes $K(u, v)$ a bounded lattice by Theorem 3.8, while (ii) (which implies $q \nless v$ ) insures that $f(u) \nless f(v)$ for the standard epimorphism (see $\S 3$ ). The third property takes care of the rank condition. Our proof will use induction on the complexity of the canonical form of $u$ and $v$.

Case 1. If $u \in X$, then $u \nless v$ implies $v \leqslant \Sigma(X \backslash\{u\})$. In this case, let $J(u, v)=$ $\{u\}$. On the other hand, if $v \in X$, then $u \nless v$ implies $u \geqslant \Pi(X \backslash\{v\})$. In this case, take $J(u, v)=\{\Pi(X \backslash\{v\})\}$. In either case, it is easy to check that properties (i)-(iii) hold. 
Case 2. If $u=\sum u_{i}$, then $u \nless v$ implies $u_{i_{0}} \nless v$ for some $i_{0}$. Let $J(u, v)=J\left(u_{i_{0}}, v\right)$, and note that conditions (i) and (ii) are preserved. Inasmuch as $D_{k}^{\prime}(\operatorname{FL}(X))=$ $(\mathscr{S P})^{k} \mathscr{S}(X)$, we have $\rho^{\prime}\left(\sum u_{i}\right)=\max \rho^{\prime}\left(u_{i}\right) \geqslant \rho^{\prime}\left(u_{i_{0}}\right)$, whence (iii) also holds.

Dually, if $v=\Pi v_{j}$, we may take $J(u, v)=J\left(u, v_{j_{0}}\right)$ for some $j_{0}$ with $u \nless v_{j_{0}}$.

Case 3. Let $u \nless v$ with $u=\Pi u_{i}$ and $v=\sum v_{i}$. Then for all $i$ we have $u_{i} \nless v$, and likewise $u \nless v_{j}$ for all $j$. Thus by induction we have sets $J\left(u_{1}, v\right)$ for each $i$, and $J\left(u, v_{j}\right)$ for each $j$, satisfying (i)-(iii). Let $J_{0}=\bigcup J\left(u_{i}, v\right) \cup \cup J\left(u, v_{j}\right)$. Let $K=$ $S_{0}\left(J_{0}\right)$, and let $f: \operatorname{FL}(X) \rightarrow K$ be the standard epimorphism.

It could happen that for some $p \in J_{0}$ we have $p \leqslant u$ and $p \nless v$. So, choose $q$ minimal in $J_{0}$ with respect to these properties. We claim that $v \leqslant \kappa(q)$. Now $K$ is a bounded lattice by Theorem 3.8, so $\kappa_{K}(q)$ exists. Since $q \nless f(v)$ but $q_{\dagger} \leqslant f(v)$, we have $f(v) \leqslant \kappa_{K}(q)$. Therefore $v \leqslant \alpha\left(\kappa_{K}(q)\right)=\kappa(q)$, as claimed. In this case, let $J(u, v)=J_{0}$. The above argument shows that (ii) holds. Condition (i) is immediate, and (iii) is not hard once we observe that $\rho^{\prime}(u)=\max \rho^{\prime}\left(u_{i}\right)+1$ and $\rho(v)=$ $\max \rho\left(v_{j}\right)+1$.

Thus we may assume that for all $p \in J_{0}, p \leqslant u$ implies $p \leqslant v$. In $K$, let $\bar{u}=f(u)$ and $\bar{v}=f(v)$. By our assumption $\bar{u} \leqslant \bar{v}$. Let

$$
q=\prod\{x \in X: x \geqslant \bar{u} \text { and } x \nless \bar{v}\} \cdot \prod\left\{\sum A: A \subseteq J_{0}, \sum A \geqslant \bar{u} \text { and } \sum A \nless \bar{v}\right\}
$$

and let $J(u, v)=J_{0} \cup\{q\}$. We need to show that $q \in J(\operatorname{FL}(X))$ and that $J(u, v)$ satisfies (i)-(iii). In fact, not surprisingly, $\mathscr{S}_{0}(J(u, v))$ will turn out to be isomorphic to the lattice obtained from $K=\mathscr{S}_{0}\left(J_{0}\right)$ by doubling the interval $\bar{v} / \bar{u}$.

First, let us show that $q \leqslant u$. Now $u=\prod u_{i}$, and for each $i$ there is a $q_{i} \in J_{0}$ with $q_{i} \leqslant u_{i}$ and $v \leqslant \kappa\left(q_{i}\right)$. So $q_{i}+\bar{u}$ is an element of the form $\sum A_{i}$ with $A_{i} \subseteq J_{0}$, which satisfies $\bar{u} \leqslant q_{i}+\bar{u} \leqslant u_{i}+u=u_{i}$, and $q_{i}+\bar{u} \nless \bar{v}$ since $q_{i}+\bar{u} \in \mathscr{S}_{0}\left(J_{0}\right)$ and $q_{i}+\bar{u}$ $\star v$. Therefore $q \leqslant \Pi\left(q_{i}+\bar{u}\right) \leqslant \Pi u_{i}=u$.

We can now see that $q$ is join irreducible in $\operatorname{FL}(X)$. Suppose $q=r+s$ with $r, s<q$. Then, by applying Whitman's condition (W) to

$\prod\{x \in X: x \geqslant \bar{u}$ and $x \nless \bar{v}\} \cdot \prod\left\{\sum A: A \subseteq J_{0}, \sum A \geqslant \bar{u}\right.$ and $\left.\sum A \nless \bar{v}\right\} \leqslant r+s$ we easily obtain that $q=\sum A_{0}$ for some $A_{0} \subseteq J_{0}, \sum A_{0} \geqslant \bar{u}$ and $\sum A_{0} \nless \bar{v}$. However, since $\sum A_{0}=q \leqslant u$, our assumption in this case would imply $\sum A_{0} \leqslant v$, whence $\sum A_{0} \leqslant \bar{v}$, a contradiction. Thus $q \in J(\mathrm{FL}(X))$.

We need to verify that $J(u, v)$ satisfies condition (C), i.e., if $q=\Pi\left(\sum q_{m n}\right) \Pi x_{k}$ canonically, then each $q_{m n} \in J_{0}$. This is equivalent to showing that $f\left(q_{m n}\right)=q_{m n}$ (where we are still using the standard epimorphism $f: \operatorname{FL}(X) \rightarrow \mathscr{S}_{0}\left(J_{0}\right)$ ). Since $f\left(q_{m n}\right) \leqslant q_{m n}$, it suffices to show that $\sum_{n} f\left(q_{m n}\right) \geqslant q$ for each $m$ (apply (W) again). However, we have $\sum_{n} f\left(q_{m n}\right) \geqslant f(q)=\bar{u}$. On the other hand, applying (W) to (the definition of $q) \leqslant \sum_{n} q_{m n}$ yields $\sum A \leqslant \sum_{n} q_{m n}$ for some $A \subseteq J_{0}$ with $\sum A \geqslant \bar{u}$ and $\sum A \nless \bar{v}$. Then $\sum A \leqslant f\left(\sum_{n} q_{m n}\right)=\sum_{n} f\left(q_{m n}\right)$, whence $\sum_{n} f\left(q_{m n}\right) \nless \bar{v}$. By the definition of $q$, we conclude that $\sum_{n} f\left(q_{m n}\right) \geqslant q$.

Next, we show that $q \nless v$. For otherwise, we could apply (W) to the inclusion $\prod\{x \in X: x \geqslant \bar{u}$ and $x \nless \bar{v}\} \cdot \prod\left\{\sum A: A \subseteq J_{0}, \sum A \geqslant \bar{u}\right.$ and $\left.\sum A \nless \bar{v}\right\} \leqslant \sum v_{j}$. If $x \leqslant \sum v_{j}$ for some $x \geqslant \bar{u}$, then $\bar{u} \leqslant x \leqslant v_{j_{0}}$ for some $j_{0}$, contrary to $J\left(u, v_{j_{0}}\right) \subseteq J_{0}$. 
If $\sum A \leqslant v$ for some $A \subseteq J_{0}$, then $\sum A \leqslant f(v)=\bar{v}$ by definition of $f$; hence no term of the second type is below $v$. But if $q \leqslant v_{j_{0}}$ for some $j_{0}$, then again $\bar{u} \leqslant v_{j_{0}}$, contrary to $J\left(u, v_{j_{0}}\right) \subseteq J_{0}$. Therefore $q \nless v$.

Let $g: \operatorname{FL}(X) \rightarrow \mathscr{S}_{0}(J(u, v))$ be the standard epimorphism. Since $\bar{u} \leqslant q \leqslant u$, we have $g(u)=q$, while $g(v)=f(v)=\bar{v}$ because $q \nless v$. It remains to show that $q$ has a lower cover in $\operatorname{FL}(X)$, and that $v \leqslant \kappa(q)$. For this it suffices to show that $\kappa_{L}(q)$ exists, where $L=\mathscr{S}_{0}(J(u, v))$, and that $g(v)=\bar{v} \leqslant \kappa_{L}(q)$.

In fact, $\bar{v}=\kappa_{L}(q)$. Now $q_{\dagger}=\bar{u}$ in $L$, because for $p \in J_{0}$ we have $p \leqslant q$ if and only if $p \leqslant f(q)=\bar{u}$. Thus $\bar{v} \geqslant q_{\dagger}$, but $\bar{v} \ngtr q$, since $v \ngtr q$. Moreover, for $\sum A \subseteq J_{0}$, $\bar{u}+A \ngtr q$ if and only if $\sum A \leqslant \bar{v}$ (using $q \nless v$ and the definition of $q$ ). Therefore $\bar{v}=\kappa_{L}(q)$.

Finally, we would like to show that the bound given for the rank of $q$ in Corollary 5.2, viz., $\rho(q) \leqslant \rho^{\prime}(u)+\rho(v)-1$, is sometimes the best possible.

Define two sequences of elements in $\operatorname{FL}(3)$ by:

$$
\begin{array}{ll}
s_{0}=z & t_{0}=x \\
s_{1}=x(y+z) & t_{1}=y+z x \\
\quad \vdots & \vdots \\
s_{2 k}=z\left(y+s_{2 k-1}\right) & t_{2 k}=x+z t_{2 k-1} \\
s_{2 k+1}=x\left(y+s_{2 k}\right) & t_{2 k+1}=y+z t_{2 k}
\end{array}
$$

Note that $\rho^{\prime}\left(s_{m}\right)=m$ and $\rho\left(t_{n}\right)=n$. It is not hard to show, using induction and Whitman's condition (W), that $s_{m} \nless t_{n}$ if $m$ is even or $n$ is odd.

THEOREM 5.3. Let $s_{m}$ and $t_{n}$ be members of the above sequences with $m>1, n>0$, and either $m$ even or $n$ odd. Let $q \in J(\mathrm{FL}(X))$ be such that $q$ has a lower cover, $q \leqslant s_{m}$ and $t_{n} \leqslant \kappa(q)$. Then $\rho(q) \geqslant m+n-1$.

Proof. We will use induction on the sum $m+n$. The induction is begun with the observation that there is no $p \in D_{0}(\mathrm{FL}(X))=\mathscr{P}(X)$ such that $p \leqslant s_{1}$ but $p \nless t_{1}$. Hence $q \leqslant s_{1}$ and $t_{1} \leqslant \kappa(q)$ imply $\rho(q) \geqslant 1$. (However, the conclusion of the theorem is false for $m=1$ and $n>1$, so we must use some care in our induction.)

Now assume $m>1, n>0$, and either $m$ even or $n$ odd, so that $s_{m} \nless t_{n}$. Let $q$ be any element of $J(\operatorname{FL}(X))$ such that $q$ has a lower cover, $q \leqslant s_{m}$ and $t_{n} \leqslant \kappa(q)$. Then the lattice $L(q)=\mathscr{S}_{0}(J(q))$ is bounded, and $f\left(s_{m}\right) \nless f\left(t_{n}\right)$ for the standard epimorphism. We wish to show that $\rho(q) \geqslant m+n-1$. There are three cases to consider.

Case 1. $m$ even, $n$ odd. In this case $s_{m}=z\left(y+s_{m-1}\right)$ and $t_{n}=y+z t_{n-1}$. Since $f\left(s_{m}\right) \nless f\left(t_{n}\right)$, we have $f\left(s_{m-1}\right) \nless f\left(t_{n}\right)$ and $f\left(s_{m}\right) \nless f\left(t_{n-1}\right)$. Thus there exist $p_{1}, p_{2}$ $\in J(q)$ such that $p_{1} \leqslant s_{m-1}, p_{1} \nless t_{n}$ and $p_{2} \leqslant s_{m}, p_{2} \nless t_{n-1}$. Taking $p_{1}$ and $p_{2}$ minimal in $J(q)$ with these properties, we obtain $t_{n} \leqslant \kappa\left(p_{1}\right)$ and $t_{n-1} \leqslant \kappa\left(p_{2}\right)$.

Now $p_{1} \neq q$, because $p_{1} \leqslant s_{m}$ would imply $p_{1} \leqslant s_{m-1} s_{m} \leqslant x z \leqslant t_{n}$, a contradiction. Likewise, $p_{2} \neq q$, or else we would have $t_{n} \leqslant \kappa\left(p_{2}\right)$, whence $p_{2} \leqslant s_{m} \leqslant x+y$ $\leqslant t_{n-1}+t_{n} \leqslant \kappa\left(p_{2}\right)$, a contradiction. Thus $\rho(q) \geqslant \max \left(\rho\left(p_{1}\right), \rho\left(p_{2}\right)\right)+1$.

If $m>2$, the inductive hypothesis implies $\rho\left(p_{1}\right) \geqslant m+n-2$. Similarly, if $n>1$ we have $\rho\left(p_{2}\right) \geqslant m+n-2$. This leaves the possibility $m=2$ and $n=1$, for which it was shown above that $\rho\left(p_{1}\right) \geqslant 1$. Thus we conclude that $\rho(q) \geqslant m+n-1$, as desired. 
Case 2. $m$ odd, $n$ odd. In this case $s_{m}=x\left(y+s_{m-1}\right)$ and $t_{n}=y+z t_{n-1}$. Since $f\left(s_{m}\right) \nless f\left(t_{n}\right)$, we have $f\left(s_{m-1}\right) \nless f\left(t_{n}\right)$, which as above gives us an element $p \in J(q)$ such that $p \leqslant s_{m-1}$ and $t_{n} \leqslant \kappa(p)$. Now $p \neq q$, because $p \leqslant s_{m}$ would imply $p \leqslant s_{m-1} s_{m} \leqslant x z \leqslant t_{n}$, a contradiction. By induction we have $\rho(p) \geqslant m+n-2$, and therefore $\rho(q) \geqslant m+n-1$.

Case 3. $m$ even, $n$ even. In this case $s_{m}=z\left(y+s_{m-1}\right)$ and $t_{n}=x+z t_{n-1}$. Thus $f\left(s_{m}\right) \nless f\left(t_{n}\right)$ implies $f\left(s_{m}\right) \nless f\left(t_{n-1}\right)$, so there is an element $p \in J(q)$ with $p \leqslant s_{m}$ and $t_{n-1} \leqslant \kappa(p)$. Again $p \neq q$, for otherwise we would have $p \leqslant s_{m} \leqslant x+y$ $\leqslant t_{n-1}+t_{n} \leqslant \kappa(p)$, a contradiction. Since $\rho(p) \geqslant m+n-2$ by induction we conclude that $\rho(q) \geqslant m+n-1$.

We conclude this section with an unpublished result of Alan Day.

THEOREM 5.4. Every finitely generated projective lattice is weakly atomic.

Proof. Let $P$ be a sublattice of $\operatorname{FL}(X)$ with $X$ finite and $f$ an endomorphism of $\operatorname{FL}(X)$ with $f(\operatorname{FL}(X))=P$ and $f^{2}=f$. Suppose $v<u$ in $P$. By Day's theorem there is a finite bounded lattice $L$ and a homomorphism $g: \mathrm{FL}(X) \rightarrow L$ with $g(v)<g(u)$. By using a homomorphic image of $L$ we may assume $g(v) \prec g(u)$ in $L$. If we let $L^{\prime}=g(P) \subseteq L$ and $h=\left.g\right|_{P}$, then $h$ is a bounded homomorphism from $P$ onto $L^{\prime}$. Moreover, $\beta_{h}(a)=f \beta_{g}(a)$. For if $g(w)=a, w \in P$, then $\beta_{g}(a) \leqslant w$. Hence $f \beta_{g}(a)$ $\leqslant f(w)=w$. Thus

$$
u \geqslant v+f \beta_{g} g(u) \succ\left(v+f \beta_{g} g(u)\right) f \alpha g(v) \geqslant v
$$

gives the desired covering in $P$.

6. The bottom of $\operatorname{FL}(n)$. A chain of $k$ covers in a lattice is a chain of $k+1$ elements such that

$$
a_{0}>a_{1}>\cdots>a_{k} .
$$

The main objective of the next four sections is to prove that, with a finite number of exceptions, covering chains in finitely generated free lattices $\operatorname{FL}(n)$ contain at most two covers. The exceptions are chains of four covers in FL(3), and of three covers in $\operatorname{FL}(n)$ for $n>3$, located at the very top and bottom of the lattice (i.e., containing either 1 or 0 ).

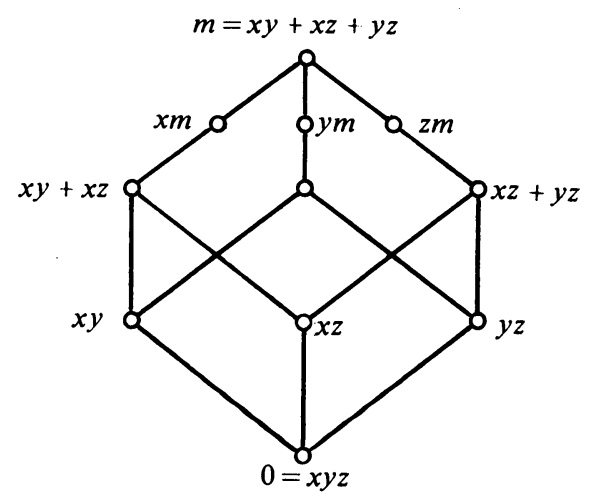

FIGURE 5 


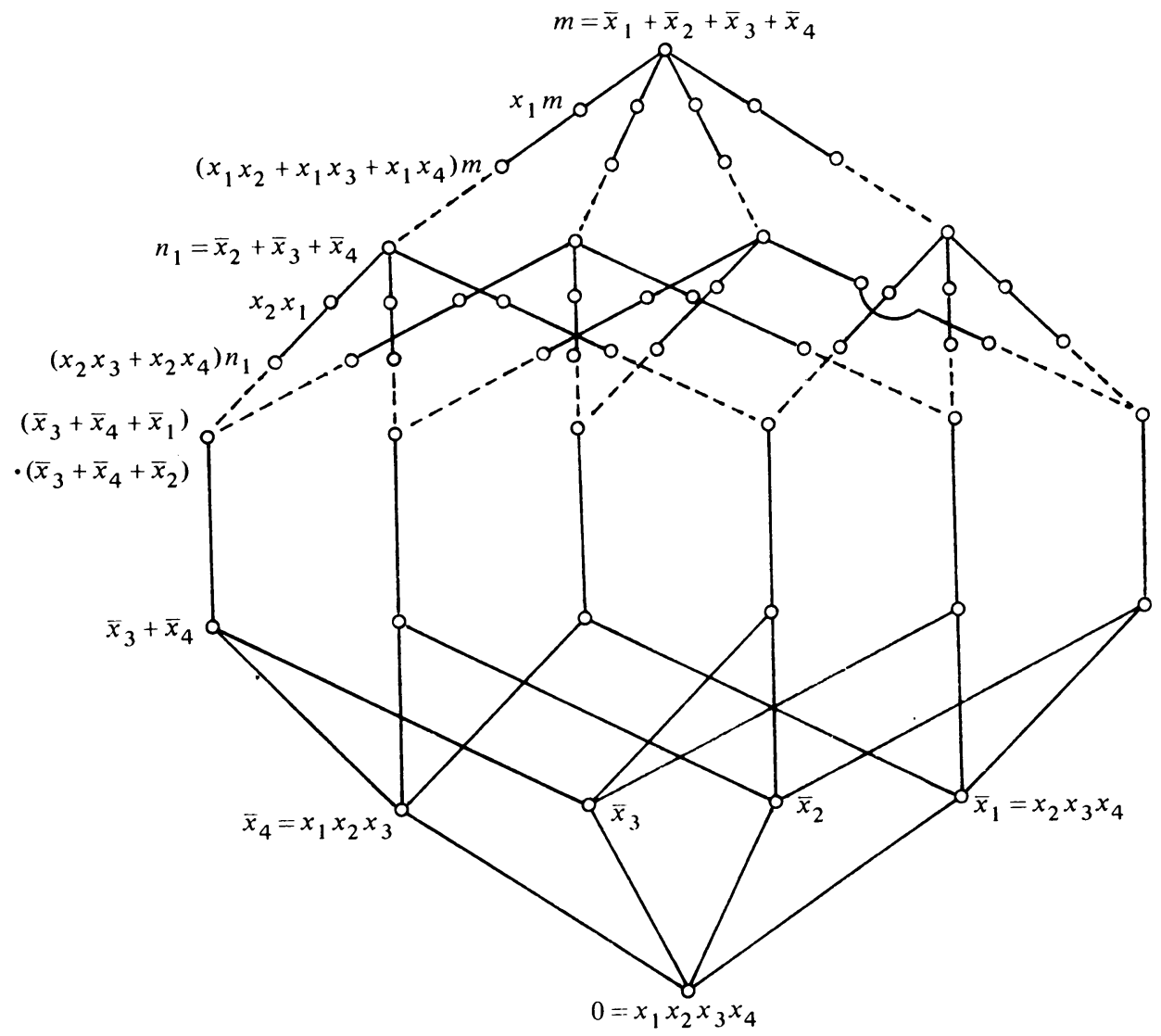

FIGURE $6(n=4)$

In the last two sections of the paper we will apply these results in an investigation of intervals $u / v$ in a free lattice. In particular, we will show that, again with finitely many exceptions at the top and bottom of the lattice, the only finite intervals in $\mathrm{FL}(n)$ are three-element chains.

Let us begin by describing some covers near the bottom of FL $(n)$; of course, the duals of all these covers also exist near the top of the lattice. Surely most, if not all, of these coverings are already known; see $[17,18]$.

THEOREM 6.1. (1) FL(3) contains the sublattice pictured in Figure 5, where every covering in the sublattice is in fact a covering in $\mathrm{FL}(3)$. No element of the sublattice covers, or is covered by, any element not in the sublattice.

(2) In $\operatorname{FL}(n)$ for $n>3$, we have the following maximal chains (see Figure 6), where $\bar{x}_{i}=\prod_{j \neq i} x_{j}$.

(a) For $i \neq j \in\{1, \ldots, n\}$,

$$
\prod_{k \neq i, j}\left(\bar{x}_{i}+\bar{x}_{j}+\bar{x}_{k}\right) \succ \bar{x}_{i}+\bar{x}_{j} \succ \bar{x}_{i} \succ 0 .
$$


(b) For $J \subseteq\{1, \ldots, n\}$ with $|J| \geqslant 3$ and $i \in J$,

$$
\sum_{j \in J} \bar{x}_{j} \succ x_{i} \sum_{j \in J} \bar{x}_{j} \succ\left[\sum_{\substack{j \neq i \\ j \in J}}\left(x_{i} x_{j}\right)\right] \sum_{j \in J} \bar{x}_{j} .
$$

Moreover, none of these elements covers, or is covered by, any element not of the indicated form.

Verification of these facts is a straightforward application of the algorithms developed in \$4. In Figure 6, solid lines indicate coverings and dotted lines indicate noncoverings. For $n \geqslant 5$, the sublattice of $\operatorname{FL}(n)$ generated by the atoms is infinite [12].

7. Totally atomic elements. An element $a$ in a lattice $L$ is totally atomic if whenever $b>a$ there is a $c \in L$ with $b \geqslant c>a$, and the dual condition holds. We will show that there are very few totally atomic elements in $\operatorname{FL}(n)$, and that they have a special form.

We need to extend the definition of $L(w)$ to include the case when $w$ is join reducible. If $w=\sum_{i=1}^{n} w_{i}$ canonically, let $J(w)=\bigcup_{i=1}^{n} J\left(w_{i}\right)$ and $L(w)=\mathscr{S}_{0}(J(w))$. Note that by Corollary 3.6, $L(w)$ is then a subdirect product of the (subdirectly irreducible) lattices $L\left(w_{i}\right)$. (However, not every lower bounded lattice is of the form $L(w)$. For example, the three-element chain $\mathscr{S}_{0}(\{x, x y\})$ is not isomorphic to $L(w)$ for any $w \in \mathrm{FL}(X)) M.(w)$ and $\mathscr{P}_{1}(M(w))$ are defined dually to $J(w)$ and $\mathscr{S}_{0}(J(w))$.

TheOrem 7.1. An element $w \in \mathrm{FL}(X)$ with $X$ finite is totally atomic if and only if $\mathscr{S}_{0}(J(w))$ and $\mathscr{P}_{1}(M(w))$ are semidistributive. Thus a join irreducible element $w \in$ $\mathrm{FL}(X)$ is totally atomic if and only if it is completely join irreducible (or zero) and each of its canonical meetands is completely meet irreducible.

Proof. Let $w$ be join irreducible with $w=\prod_{i=1}^{n} w_{i}$ canonically. Clearly the two stated conditions for $w$ to be totally atomic are equivalent, and by Theorems 2.3 and 4.1(2), if $w$ is totally atomic then $w$ satisfies these conditions. So suppose $w$ is completely join irreducible and each $w_{i}$ is completely meet irreducible. Let $v_{i}=$ $\kappa^{\prime}\left(w_{i}\right)+w$. Then $w \prec v_{i} \leqslant \prod_{j \neq i} w_{j}$ and, since $w=\prod_{i=1}^{n} w_{i}$ canonically, if $u v_{i}=w$ then $u \leqslant w_{i}$. Suppose $w<u$. If $v_{i} \nless u$, then $v_{i} u=w$ and hence $u \leqslant w_{i}$. Thus, either there is an $i$ with $v_{i} \leqslant u$, or else $u \leqslant \prod_{i=1}^{n} w_{i}=w$. But the latter is a contradiction. Therefore, $w$ is totally atomic.

For $u \in \operatorname{FL}(X)$ let $\sigma_{u} \in \operatorname{End}(\operatorname{FL}(X))$ be defined by $\sigma_{u}(x)=u+x$ for $x \in X$. $\mu_{u}$ is defined dually. Let $G$ be the smallest subset of $\operatorname{FL}(X)$ containing $X$ and such that if $w \in G$ and $x \in X-\operatorname{var}(w)$, then $\sigma_{x}(w)$ and $\mu_{x}(w) \in G$. We shall show that the set of totally atomic elements of $\operatorname{FL}(X)$ is $G$.

If $w \in G$ is join irreducible and $w \notin X$, then we can write

$$
w=\mu_{p_{n+1} \sigma_{s_{n}}} \mu_{p_{n}} \cdots \mu_{p_{2}} \sigma_{s_{1}}\left(y_{1} \cdots y_{m}\right),
$$

where $m>1, s_{i}=\sum_{j} s_{i j}$ with $s_{i j} \in X$, and $p_{i}=\prod_{j} p_{i j}$ with $p_{i j} \in X$. We allow $p_{n+1}=1$ so that, in this case, $\mu_{p_{n+1}}=\mu_{1}$ is the identity, and we also allow $n=0$ (in which event we take $p_{1}=1$ ) so that $w=y_{1} \cdots y_{m}$ is a possibility. 
For example, if $X=\{x, y, z\}$, then $G$ contains only the following join irreducible elements of $\operatorname{FL}(X)$ and their images under automorphisms of $\operatorname{FL}(X)$, i.e., permutations of the variables.

(1) $x$.

(2) $x y$.

(3) $x y z$.

(4) $\sigma_{z}(x y)=(x+z)(y+z)$.

With $X^{\prime}=\{x, y, z, t\}$ we add to the above list all elements of the following forms:

(5) $x y z t$.

(6) $\sigma_{z+t}(x y)=(x+z+t)(y+z+t)$.

(7) $\sigma_{t}(x y z)=(x+t)(y+t)(z+t)$.

(8) $\mu_{t} \sigma_{z}(x y)=(x t+z t)(y t+z t)$.

Of course, the duals of these words give us meet irreducible elements in $G$.

In particular, note that $G$ is finite-in fact, if $|X|=n$, then $|G| \leqslant n ! 2^{n-2} \sqrt{e}$.

LEMMA 7.2. When the expression (*) for $w \in G$ is written out, $w$ is in canonical form.

Proof. It was shown by Whitman [17] that a word $u=\prod_{i=1}^{n}\left(\sum_{j=1}^{m_{i}} u_{i j}\right) \prod_{k=1}^{p} x_{k}$ is in canonical form if and only if

(i) each $u_{i}=\sum_{j} u_{i j}$ is in canonical form,

(ii) $\left\{u_{i}: i=1, \ldots, n\right\} \cup\left\{x_{k}: k=1, \ldots, p\right\}$ is an antichain, and

(iii) for every $i, j, u_{i j} \ngtr u$.

It is easy to see that these properties are preserved under $\sigma_{x}$ and $\mu_{x}$ whenever $x \notin \operatorname{var}(u)$, from which the lemma follows.

Lemma 7.3. Let $w \in G$ have the form (*). Then

(1) $J(w)=\{w\} \cup\left\{\mu_{p_{n+1}} \sigma_{s_{n}} \cdots \mu_{p_{t}}(z): z=y_{i}\right.$ for some $i$, or $z=s_{k j}$ or $p_{k j}$ for some $k<t$, and $t=2, \ldots, n+1\}$.

(2) $w_{\dagger}=\mu_{p_{n+1}} \sigma_{s_{n}} \cdots \mu_{p_{2}} \sigma_{s_{1}}(0)$, where $w_{\dagger}$ denotes the lower cover of $w$ in $L(w)$.

(3) $K(w)=\left\{\mu_{p_{n+1}} \sigma_{s_{n}} \cdots \mu_{p_{t}}(z): z=s_{k j}\right.$ for some $k<t$ and some $j$, and $t=$ $2, \ldots, n+1\}$.

(4) $\kappa_{L(w)}(w)=\mu_{p_{n+1}}\left(s_{1}+\cdots+s_{n}\right)$.

Proof. (1) Let $\tau$ denote the endomorphism $\mu_{p_{n+1} \sigma_{s_{n}}} \cdots \sigma_{s_{2}} \mu_{p_{2}}$. Then we calculate

$$
\begin{aligned}
w & =\tau \sigma_{s_{1}}\left(\prod_{i} y_{i}\right)=\tau \prod_{i}\left(y_{i}+s_{11}+\cdots+s_{1 q}\right) \\
& =\prod_{i}\left(\tau\left(y_{i}\right)+\tau\left(s_{11}\right)+\cdots+\tau\left(s_{1 q}\right)\right),
\end{aligned}
$$

whence $\tau\left(y_{i}\right)$ and $\tau\left(s_{1 j}\right)$ are in $J(w)$. Now (1) follows easily by induction.

(2) Clearly $\mu_{p_{n+1} \sigma_{s_{n}}} \cdots \mu_{p_{2} \sigma_{s_{1}}}(0)<w$. We need to know when $u=\mu_{p_{n+1}} \sigma_{s_{n}} \cdots$ $\mu_{p_{t}}(z) \in J(w)$ is below $w$. Since $\sigma_{x}$ and $\mu_{x}$ are one-to-one on $\operatorname{FL}(X-\{x\})$, this is equivalent to $z \leqslant \sigma_{s_{t-1}} \mu_{p_{t-1}} \cdots \sigma_{s_{1}}\left(\Pi_{i} y_{i}\right)$. If $z=s_{t-1, j}$ for some $j$, then clearly the inequality holds. But if $z=s_{k j}$ for some $k<t-1$, or if $z=p_{k j}$ for some $k$ or $z=y_{i}$ for some $i$, set $z=1$ and all other variables to 0 . Then the right-hand side $\sigma_{s_{t-1}} \mu_{p_{t-1}} \cdots \sigma_{s_{1}}\left(\Pi_{i} y_{i}\right)$ evaluates to 0 (there are two cases, depending on whether or not $p_{t-1}=z$ ), while $z=1$, so the inclusion fails. Thus, $u \in J(w)$ is strictly below $w$ if and only if $u=\mu_{p_{n+1} \sigma_{n}} \sigma_{s} \cdots \mu_{p_{t}}\left(s_{t-1, j}\right)$ for some $t$ with $2 \leqslant t \leqslant n+1$ and some $j$. 
Now if $t>2$ and $s^{\prime}=\sum_{k \neq j} s_{t-1, k}$ (possibly zero), then

$$
\begin{aligned}
\mu_{p_{n+1}} \sigma_{s_{n}} \cdots \mu_{p_{t}}\left(s_{t-1, j}\right) & =\mu_{p_{n+1}} \sigma_{s_{n}} \cdots \mu_{p_{t}} \sigma_{s_{t-1, j}}(0) \\
& \leqslant \mu_{p_{n+1}} \sigma_{s_{n}} \cdots \mu_{p_{t}} \sigma_{s_{t-1, j}}\left(\sigma_{s^{\prime}} \mu_{p_{t-1}}\left(s_{t-2, m}\right)\right) \\
& =\mu_{p_{n+1}} \sigma_{s_{n}} \cdots \mu_{p_{t-1}}\left(s_{t-2, m}\right) .
\end{aligned}
$$

Therefore

$$
w_{\dagger}=\sum\{u \in J(w): u<w\}=\sum_{j} \mu_{p_{n+1} \sigma_{s_{n}}} \cdots \mu_{p_{2}}\left(s_{1 j}\right)=\mu_{p_{n+1}} \sigma_{s_{n}} \cdots \mu_{p_{2}} \sigma_{s_{1}}(0)
$$

as claimed.

(3), (4) Now let $u$ be an element of $J(w)$ with $w \nless u$ but $w \leqslant w_{\dagger}+u$. As above, if $u=\mu_{p_{n+1}} \sigma_{s_{n}} \cdots \mu_{p_{t}}(z)$, then (using (2)) $w \leqslant w_{\dagger}+u$ is equivalent to

$$
\sigma_{s_{t-1}} \mu_{p_{t-1}} \cdots \sigma_{s_{1}}\left(\prod_{i} y_{i}\right) \leqslant \sigma_{s_{t-1}} \mu_{p_{t-1}} \cdots \sigma_{s_{1}}(0)+z \text {. }
$$

If we set all $y_{i}=1$, all $p_{i j}=1$, and all $s_{i j}=0$, then the left-hand side of $(* *)$ evaluates to 1 and the right-hand side is $z$. Thus, $u \notin K(w)$ implies $z=y_{i}$ for some $i$ or $z=p_{k j}$ for some $k, j$. Hence

$$
\begin{aligned}
K(w) \subseteq\left\{\mu_{p_{n+1} \sigma_{n}} \cdots\right. & \mu_{p_{t}}(z): z=s_{k j} \\
& \text { for some } k<t \text { and } t=2, \ldots, n+1\} .
\end{aligned}
$$

Call the right-hand side of $(* * *) R$. Clearly, for each $u \in R$ we have

$$
u=\mu_{p_{n+1} \sigma_{s_{n}}} \cdots \mu_{p_{t}}\left(s_{k j}\right) \leqslant \mu_{p_{n+1}}\left(s_{1}+\cdots+s_{n}\right)=\sum_{k} \sum_{j} \mu_{p_{n+1}}\left(s_{k j}\right) \in \mathscr{S}(R) .
$$

Therefore $\mu_{p_{n+1}}\left(s_{1}+\cdots+s_{n}\right)=\sum R$. On the other hand, by setting each $s_{k j}=0$, each $p_{k j}=1$ and each $y_{i}=1$, we conclude that $w \nless \mu_{p_{n+1}}\left(s_{1}+\cdots+s_{n}\right)$. Thus by Theorem 4.3 we have $\kappa_{L(w)}(w)=\mu_{p_{n+1}}\left(s_{1}+\cdots+s_{n}\right)$, so in particular, $w$ has a lower cover in $\operatorname{FL}(X)$. It also follows that we must have equality in $(* * *)$.

Lemma 7.3 shows that every join irreducible element in $G$ is completely join irreducible. The canonical meetands of an element of the form (*) are of the form $\mu_{p_{n+1} \sigma_{s_{n}}} \cdots \mu_{p_{2}}\left(y_{i}+\sum_{j} s_{1 j}\right)$. By the dual argument each of these elements is completely meet irreducible. Therefore by Theorem 7.1 we may conclude that:

COROLlaRY 7.4. Each $w \in G$ is totally atomic.

To prove the converse, we will need the following

LEMMA 7.5. Let $u \in G$ be meet irreducible with $u=u_{1}+\cdots+u_{n}$ canonically, and let $x \in X$. If $u_{1} \nless x$ but $u_{i} \leqslant x$ for $2 \leqslant i \leqslant n$, then $x u \in \mathscr{P}(X)$.

Proof. Clearly we may assume $u \notin X$, so that $n \geqslant 2$. If $u=\sum_{i=1}^{n} y_{i}$ with $y_{i} \in X$, then $y_{i} \leqslant x$ for $2 \leqslant i \leqslant n$ implies $n=2$ and $y_{2}=x$, whence $u=y_{1}+x$ and $x u=x$. Thus, since $u \in G$, we may assume $u=\rho_{x_{k}} \cdots \rho_{x_{1}}\left(y_{1}+\cdots+y_{n}\right)$, where $x_{1}, \ldots, x_{k}$, $y_{1}, \ldots, y_{k}$ are distinct members of $X, \rho_{x_{1}}=\mu_{x_{1}}$, and $\rho_{x_{i}}=\sigma_{x_{i}}$ or $\mu_{x_{i}}$ for $i=2, \ldots, k$.

Suppose $\rho_{x_{i}}=\sigma_{x_{i}}$ for some $i$ with $1<i \leqslant k$. Then choosing $r$ maximal such that $\rho_{x_{r}}=\sigma_{x_{r}}$ we have

$$
u=\mu_{x_{k}} \cdots \mu_{x_{r+1} \sigma_{x_{r}}} \cdots \mu_{x_{1}}\left(y_{1}+\cdots+y_{n}\right)
$$


Moreover, $x \notin\left\{x_{k}, x_{k-1}, \ldots, x_{r+1}\right\}$ as $u \nless x$. However, for $i \geqslant 2$, we now have

$$
x \geqslant u_{i}=\mu_{x_{k}} \cdots \mu_{x_{r}, 1} \sigma_{x_{r}} \cdots \mu_{x_{1}}\left(y_{i}\right) \text {. }
$$

If we set $x=0$ and $z=1$ for $z \in X-\{x\}$, then $u_{i}$ evaluates to 1 (there are two cases, depending on whether or not $x=x_{r}$ ), which for $i \geqslant 2$ gives the contradiction $0 \geqslant 1$. Therefore $\rho_{x_{i}}=\mu_{x_{i}}$ for $1 \leqslant i \leqslant k$.

So now we have $u_{i}=x_{k} \cdots x_{1} y_{i}$. As $u_{1} \nless x$ and $u_{i} \leqslant x$ for $2 \leqslant i \leqslant n$, this implies $y_{2}=x$ and $n=2$. Thus

$$
x u=x\left(x_{k} \cdots x_{1} y_{1}+x_{k} \cdots x_{1} x\right)=x_{k} \cdots x_{1} x \in \mathscr{P}(X)
$$

as desired.

We want to show that if $w \in \operatorname{FL}(X)$ is totally atomic, then $w \in G$. Certainly this is true if $w \in X \cup \mathscr{P}(X) \cup \mathscr{S}(X)$. So if say $w=w_{1} \cdots w_{m}$ canonically, then we may assume inductively that $m>1$ and each $w_{i} \in G$. (If $w$ is totally atomic and $u \in J(w) \cup M(w)$, then $u$ is totally atomic by Theorem 7.1.) Moreover, since $w$ is completely join irreducible, Theorem 4.4 applies to say that for every $i$, if say $w_{i}=\sum_{j} w_{i j}$ canonically, then for all but one $j$ we have $w_{i j} \leqslant w \leqslant w_{t}$ for all $t$. Thus the following lemma applies to $w$ to yield the desired result.

Lemma 7.6. Let $w=\prod_{i=1}^{m} w_{i}$ be an irredundant meet, where each $w_{i}$ is meet irreducible and in G. Let $w_{i}=\sum_{j} w_{i j}$ canonically (if $\left.w_{i} \in X, w_{i}=w_{i 1}\right)$. Assume

$$
\forall i \forall j>1 \forall t: w_{i j} \leqslant w_{t} \text {. }
$$

Then $w \in G$.

Proof. If $w_{i} \in X$ for any $i$, then we are done by Lemma 7.5, for then $w \in \mathscr{P}(X)$ $\subseteq G$. Thus we may assume

$$
w_{i}=\rho_{x_{i k}}^{i} \cdots \rho_{x_{i 1}}^{i}\left(y_{i 1}+\cdots+y_{i n_{i}}\right)
$$

where $n_{i} \geqslant 2$, each $\rho$ in either $\sigma$ or $\mu$, and $k_{i}=0$ is possible (so $w_{i}=y_{i 1}+\cdots+y_{i n_{i}}$ in this case) but $\rho_{x_{i 1}}^{i}=\mu_{x_{i 1}}$ if $k_{i} \geqslant 1$.

First, let us consider the case when $k_{i}=0$ for every $i$. For every pair $i \neq i^{\prime}$, we have $\sum_{j>1} y_{i j} \leqslant w_{i^{\prime}}=\sum_{j=1}^{n_{i^{\prime}}=1} y_{i^{\prime} j}$ but $y_{i 1} \nless w_{i^{\prime}}$ (as $w_{i} \nless w_{i^{\prime}}$ ), so $\left\{y_{i 2}, \ldots, y_{i n_{i}}\right\}=$ $\left\{y_{i^{\prime} 2}, \ldots, y_{i^{\prime} n_{i}}\right\}$. Therefore $w=\sigma_{y_{12}} \cdots \sigma_{y_{1 n_{1}}}\left(\prod_{i=1}^{m} y_{i 1}\right) \in G$, as desired.

Thus we may assume that $k_{1} \geqslant 1$, and fix $x_{1}=x_{1 k_{1}}$, so that $w_{1}=\rho_{x_{1}}^{1} w_{1}^{\prime}$.

Claim. Either (a) for every $i, w_{i}=\sigma_{x_{1}} w_{i}^{\prime}$, where $w_{1}^{\prime} \in G \cap \operatorname{FL}\left(X-\left\{x_{1}\right\}\right)$, or (b) for every $i, k_{i} \geqslant 1$ and $w_{i}=\mu_{x_{1}} w_{i}^{\prime}$, where $w_{i}^{\prime} \in G \cap \operatorname{FL}\left(X-\left\{x_{1}\right\}\right)$.

To prove the claim, we first consider the possibility that $k_{i}=0$ for some $i$, so that $w_{i}=y_{i 1}+\cdots+y_{i n_{i}}$. If $w_{1}=\mu_{x_{1}} w_{1}^{\prime}$, then $y_{i 2} \leqslant w_{1} \leqslant x_{1}$ whence $y_{i 2}=w_{1}=x_{1}$, contrary to our assumption that $k_{1} \geqslant 1$. Thus $w_{1}=\sigma_{x_{1}} w_{1}^{\prime}$, and from $x_{1} \leqslant w_{12} \leqslant w_{i}=$ $\sum_{j} y_{i j}$ it follows that $y_{i j}=x_{1}$ for some $j$. So in this case we can write $w_{i}=\sigma_{x_{1}}\left(\sum_{j^{\prime} \neq j} y_{i j^{\prime}}\right)$. If $k_{i}=0$ for every $i>1$, (a) of the claim holds.

Now assume $k_{1} \geqslant 1$ and $k_{2} \geqslant 1$. First suppose $\rho_{x_{1 k_{1}}}^{1}=\mu_{x_{1}}$ and $\rho_{x_{2 k_{2}}}^{2}=\sigma_{x_{2}}$. Then $w_{1} \leqslant x_{1}$ and $w_{2} \geqslant x_{2}$, whence $x_{1} \neq x_{2}$. However, by hypothesis we have $w_{22} \leqslant w_{1}$, which implies

$$
x_{2} \leqslant \sigma_{x_{2}} \cdots \rho_{x_{21}}^{2}\left(y_{22}\right)=w_{22} \leqslant w_{1} \leqslant x_{1}
$$


a contradiction. So either all $w_{i}$ with $k_{i}>1$ begin with a $\sigma$, or else they all begin with a $\mu$.

Now assume $w_{1}$ begins with $\sigma_{x_{1}}$ and $w_{2}$ with $\sigma_{x_{2}}$, and suppose $x_{1} \neq x_{2}$. Then we can write $w_{2}=\sigma_{x_{2}} \sigma_{z_{1}} \cdots \sigma_{z_{r}} \mu_{z_{r+1}} \cdots\left(\sum_{j} y_{2 j}\right)$, where $\mu_{z_{r+1}}$ is the first $\mu$ occurring in the expression for $w_{2}$. If $x_{1} \in\left\{z_{1}, \ldots, z_{r}\right\}$, then since the $\sigma_{z}$ 's commute we have $w_{2}=\sigma_{x_{1}} w_{2}^{\prime}$, as claimed. Otherwise, set $x_{1}=1$ and $z=0$ for $z \in X-\left\{x_{1}\right\}$. Then $w_{12}$ evalutes to 1 , but $w_{2}$ evaluates to 0 (again there are two cases depending on whether $x_{1}=z_{r+1}$ ), contrary to $w_{12} \leqslant w_{2}$. A similar argument applies when both begin with $\mu$, proving the claim.

So now we know that either $w_{i}=\sigma_{x_{1}} w_{i}^{\prime}$ for all $i$, or $w_{i}=\mu_{x_{1}} w_{i}^{\prime}$ for all $i$, where each $w_{i}^{\prime}$ is either a variable or still a sum. Hence each $w_{i}^{\prime}$ is meet irreducible, and clearly $w_{i}^{\prime} \in G \cap \operatorname{FL}\left(X-\left\{x_{1}\right\}\right)$. In case (a), let $\varphi: \operatorname{FL}(X) \rightarrow \operatorname{FL}\left(X-\left\{x_{1}\right\}\right)$ be defined by $\varphi\left(x_{1}\right)=0$ and $\varphi(y)=y$ for all $y \in X-\left\{x_{1}\right\}$. If (b) holds, then let $\varphi\left(x_{1}\right)=1$ and $\varphi(y)=y$ for $y \neq x$. In either case, $\varphi\left(w_{i}\right)=w_{i}^{\prime}$ and $\rho_{x_{1}}\left(w_{i}^{\prime}\right)=w_{i}$ for the appropriate $\rho$. Therefore $w^{\prime}=\varphi(w)=w_{1}^{\prime} \cdots w_{m}^{\prime}$ is an irredundant meet, each $w_{i}^{\prime}=\sum_{j} w_{i j}^{\prime}$ canonically and

$$
\forall i \forall j>1 \forall t: w_{i j}^{\prime} \leqslant w_{t}^{\prime} .
$$

Hence by induction $w^{\prime} \in G \cap \operatorname{FL}\left(X-\left\{x_{1}\right\}\right)$. But then $w=\rho_{x_{1}} w^{\prime} \in G$.

Combining everything we have done in this section so far, we obtain the desired characterization of totally atomic elements.

TheOrem 7.7. An element $w \in \mathrm{FL}(X)$ with $X$ finite is totally atomic if and only if $w \in G$.

8. Lemmas on totally atomic elements. In this section we will prove some lemmas about totally atomic elements which will be used in our investigation of covering chains in $\mathrm{FL}(X)$. The first lemma shows why totally atomic elements play an important role in covering chains.

LEMMA 8.1. Let $u$ be a completely meet irreducible element of $\operatorname{FL}(X)$, where $X$ is finite, and let $u \succ v$. Let $u_{1}$ be the unique member of $\mathrm{CJ}(u)$ such that $u_{1} \nless v$. Then $u_{1}$ is totally atomic.

Proof. Note that $u_{1} \in J(\operatorname{FL}(X))$. By Theorem 2.3, $u_{1}$ has a lower cover $u_{1 *}$, so every member of $J\left(u_{1}\right)$ is completely join irreducible. On the other hand, if $u_{1}=\Pi_{j} u_{1 j}$ canonically, then $M\left(u_{1}\right)=\bigcup_{j} M\left(u_{1 j}\right) \subseteq M(u)$, so every element in $M\left(u_{1}\right)$ is completely meet irreducible. By Theorem $7.1, u_{1}$ is totally atomic.

Our next two lemmas are more technical.

LEMMA 8.2. Let $w$ be a join irreducible totally atomic element of $\operatorname{FL}(X)$ (with $X$ finite). Then $\kappa(w)^{*}=w+\kappa(w)$ is not completely meet irreducible.

Proof. Let $w$ be a totally atomic join irreducible element of $\operatorname{FL}(X)$. If $w \in X$, then $w+\kappa(w)=1$, which is by convention not meet irreducible. Hence we may assume $w=\prod_{i=1}^{n} w_{i}$ with $n>1$. Since $w$ has the form (*), there are automorphisms of $\operatorname{FL}(X)$ interchanging the $w_{i}$ 's, but leaving $w$, and hence $\kappa(w)$, fixed. So no $w_{i}$ is 
above $\kappa(w)$ unless $w \geqslant \kappa(w)$, i.e., $\kappa(w)=w_{*}$. Now if $\kappa(w)=w_{*}$, then $w+\kappa(w)=w$ is join irreducible and not a variable, and hence not (completely) meet irreducible. Thus we may assume that no $w_{i}$ is above $\kappa(w)$.

Next, observe that $w$ is a canonical joinand of $\kappa(w)^{*}$. Indeed, in the proof of Theorem 2.1 we saw that $\kappa(w)$ is a canonical meetand of $w_{*}$, and this is just the dual of that statement. Thus, by the dual of Theorem 4.4, if $w+\kappa(w)$ were completely meet irreducible we would have $w_{i} \geqslant \kappa(w)^{*}$ for all but one $i$, contrary to the preceding paragraph. We conclude that $w+\kappa(w)$ is not completely meet irreducible.

COROllaRY 8.3. Let $u$ be a completely meet irreducible element of $\operatorname{FL}(X)$, where $X$ is finite. If $u \succ v$, then $v$ is meet reducible.

Proof. By the proof of Theorem 2.3, whenever $u$ is meet irreducible and $u \succ v$ then there is a unique member $u_{1}$ of $\mathrm{CJ}(u)$ such that $u_{1} \nless v$, and in fact $v=u \kappa\left(u_{1}\right)$. Thus $v$ will be meet reducible except when $v=\kappa\left(u_{1}\right)$. Applying Lemma 8.1 to our situation, since $u$ is completely meet irreducible, $u_{1}$ is totally atomic. By Lemma 8.2, $u_{1}+\kappa\left(u_{1}\right)$ is not completely meet irreducible. However, $u_{1}+v=u$ is completely meet irreducible, so we conclude that $v \neq \kappa\left(u_{1}\right)$. Hence $v$ is meet reducible.

LEMMA 8.4. If $w$ is a join irreducible totally atomic element of $\mathrm{FL}(X)$ (with $X$ finite) and $w \notin \mathscr{P}(X)$, then $\kappa(w)$ is not totally atomic.

Proof. Again we use the representation

$$
w=\mu_{p_{n+1} \sigma_{s_{n}}} \cdots \mu_{p_{2}} \sigma_{s_{1}}\left(y_{1} \cdots y_{m}\right),
$$

and since $w \notin \mathscr{P}(X)$ we are assuming $n \geqslant 1$. By Lemma 7.3(1), $J(w)$ contains either $\mu_{p_{2} \sigma_{s_{1}}}\left(\prod_{i=1}^{m} y_{i}\right)$ (if $\left.n=1\right)$ or $\mu_{p_{n+1} \sigma_{s_{n}}}\left(\mu_{p_{n}} y_{1}\right)$ (if $n>1$ ). In either event, $J(w)$ contains an element of the form $u=\mu_{p} \sigma_{s}\left(\prod_{k=1}^{M} z_{k}\right)$, where $M>1$, each $z_{k} \in X, s \in \mathscr{S}(X)$ and $p \in \mathscr{P}(X) \cup\{1\}$, and the variables are distinct. By the observation preceding Theorem 4.6, $\kappa(u) \in M(\kappa(w))$ (i.e., $\kappa(u)=\alpha(v)$ for some meet irreducible element $v$ of $L(w))$.

Let $p=\Pi P$, where $P \subseteq X$ (if $p=1$, then $P=\varnothing$ ). In the proof of Corollary 4.2, we observed (without the notation) that if $q \in J(\operatorname{FL}(X))$ has a lower cover and $X \cap Y=\varnothing$, then $\kappa_{\mathrm{FL}(X \cup Y)}(q)=\sigma_{\sum Y}\left(\kappa_{\mathrm{FL}(X)}(q)\right)$. Since $\kappa$ is a bijection, another way of putting this is that if $t$ is completely meet irreducible and $t=\sigma_{\sum Y} t^{\prime}$, where $\operatorname{var}\left(t^{\prime}\right) \cap Y=\varnothing$, then $\kappa_{\mathrm{FL}(X)}^{\prime}(t)=\kappa_{\mathrm{FL}(X-Y)}^{\prime}\left(t^{\prime}\right)$. The dual of this observation applies here to show that $\kappa(u)=\kappa_{\mathrm{FL}(X)}(u)=\kappa_{\mathrm{FL}(X-P)}\left(\sigma_{s}\left(\prod_{k=1}^{M} z_{k}\right)\right)$.

Applying the algorithm of Theorem 4.6, we thus find that $\kappa(u)=s+$ $\sum_{j=1}^{N} \bar{s}_{j}\left(\prod_{k=1}^{M} \bar{z}_{k}\right)$, where $\bar{x}=\sum(X-P-\{x\})$. Now if $s \in X$, then this is already in canonical form $\left(\kappa(u)=s+\bar{s}_{1} \cdots \bar{z}_{M}\right)$. Otherwise (i.e., if $s=\sum s_{j}$ with $N>1$ ), the canonical form is $\kappa(u)=\sum_{j=1}^{N} \bar{s}_{j}\left(\prod_{k=1}^{M} \bar{z}_{k}\right)$. Either way, we find that $\bar{s}_{1} \bar{z}_{1} \cdots \bar{z}_{M}$ is a canonical summand, whence $\bar{s}_{1} \bar{z}_{1} \cdots \bar{z}_{M} \in J(\kappa(u))$, and therefore $\bar{s}_{1} \bar{z}_{1} \cdots \bar{z}_{M}$ $\in J(\kappa(w))$. (It is straightforward to prove that if $t \in M(v)$ and $t \notin X$, then $J(t) \subseteq J(v)$-use induction on the complexity of $v$.) But an easy application of Theorem 4.4 shows that $\bar{s}_{1} \bar{z}_{1} \cdots \bar{z}_{M}$ fails to have a lower cover. Therefore $\kappa(w)$ is not totally atomic. 
Our next result stands in interesting contrast with Day's theorem, for it shows that there are intervals in $\mathrm{FL}(X)$ which contain no completely join irreducible element.

THEOREM 8.5. Let $w$ be a completely join irreducible element of $\operatorname{FL}(X)$ (with $X$ finite) such that $\kappa(w)$ is not totally atomic. Then there is no completely join irreducible element $p \in \mathrm{FL}(X)$ with $w_{*} \leqslant p \leqslant \kappa(w)$.

Proof. To begin with assume only that $w$ is completely join irreducible in $\operatorname{FL}(X)$. Note that if $u \in J(w)-\{w\}$ and $u \leqslant w+\kappa(w)$, then $u \leqslant \kappa(w)$. To see this, let $g$ : $\operatorname{FL}(X) \rightarrow L(w) / \operatorname{con}\left(w, w_{\dagger}\right)=\mathscr{S}_{0}(J(w)-\{w\})$ be the standard epimorphism. Then $u \leqslant \kappa(w)+w$ implies $u=g(u) \leqslant g(\kappa(w)+w)=g(\kappa(w)) \leqslant \kappa(w)$.

Now let $w=\Pi_{i} w_{i}$ canonically. We claim that if $w \notin X$, then for all $i$ we have $w_{i} \nless w+\kappa(w)=\kappa(w)^{*}$. For if $w_{i} \in X$, then $w_{i} \leqslant w+\kappa(w)$ implies $w_{i} \leqslant w$ or $w_{i} \leqslant \kappa(w)$. But $w_{i} \leqslant w$ implies $w=w_{i} \in X$, contrary to assumption, and $w_{i} \leqslant \kappa(w)$ implies $w \leqslant \kappa(w)$, a contradiction. On the other hand, if $w_{i}=\Sigma_{j} w_{i j}$, then $w_{i j} \in$ $J(w)-\{w\}$ for all $j$. Hence, by the observation of the preceding paragraph, $w_{i}=\sum_{j} w_{i j} \leqslant w+\kappa(w)$ implies $w_{i} \leqslant \kappa(w)$, whence $w \leqslant \kappa(w)$, again a contradiction. Thus $w_{i} \nless w+\kappa(w)$ for all $i$.

Now let us add the hypothesis that $\kappa(w)$ is not totally atomic. Supposing that there exists a completely join irreducible element in $\kappa(w) / w_{*}$, let $p$ be such an element of minimal complexity. If $p \in X$, then $w_{*}=w \kappa(w) \leqslant p$ implies either $w \leqslant p$ or $\kappa(w) \leqslant p$. Now $w \leqslant p$ is out because $p \leqslant \kappa(w)$. If $\kappa(w) \leqslant p$, then $\kappa(w)=$ $p \in X$, contrary to our assumption that $\kappa(w)$ is not totally atomic. Hence $p \notin X$.

Now let $p=\Pi_{i}\left(\sum_{j} p_{i j}\right) \cdot \Pi_{k} x_{k}$, and let $\kappa(w)=\sum_{m} u_{m}$ be the canonical join representation of $\kappa(w)$. Since $p \leqslant \kappa(w)$, we may apply (W) to the inclusion

$$
\prod_{i}\left(\sum_{j} p_{i j}\right) \cdot \prod_{k} x_{k} \leqslant \sum_{m} u_{m} .
$$

If $x_{k} \leqslant \kappa(w)$ for some $k$, then $w_{*} \leqslant p \leqslant x_{k} \leqslant \kappa(w)$, which is out as above. If $p \leqslant u_{m}$ for some $m$, we obtain $w_{*} \leqslant u_{m}$. This situation is the dual of $w_{i} \leqslant \kappa(w)^{*}$, which was shown earlier not to occur.

Thus there must be an $i$ such that $\sum_{j} p_{i j} \leqslant \kappa(w)$. Fixing this $i$, note that $w_{*} \leqslant p \leqslant \sum_{j} p_{i j}$, so we can apply (W) to the inclusion

$$
w \kappa(w) \leqslant \sum_{j} p_{i j} .
$$

Now $w \nless \sum_{j} p_{i j}$ because $\sum_{j} p_{i j} \leqslant \kappa(w)$. If $w_{*} \leqslant p_{i j}$ for some $j$, then $w_{*} \leqslant p_{i j} \leqslant \kappa(w)$ and $p_{i j}$ has a lower cover because $p_{i j} \in J(p)$, contradicting the minimal complexity of $p$. We conclude that $\kappa(w) \leqslant \sum_{j} p_{i j}$, whence in fact $\kappa(w)=\sum_{j} p_{i j}$. Because the expression for $p$ was canonical, this one is also.

Now $\kappa(w)$ is always completely meet irreducible, and hence so is every member of $M(\kappa(w))$. On the other hand, $J(\kappa(w))=\bigcup_{j} J\left(p_{i j}\right)$, and because $p$ was completely join irreducible, so is every element in every $J\left(p_{i j}\right)$. We conclude then by Theorem 7.1 that $\kappa(w)$ is totally atomic, contrary to the hypothesis. Therefore there is no completely join irreducible $p$ in $\kappa(w) / w_{*}$. 
9. Chains of covers. In this chapter we will prove our main result about covering chains in $\mathrm{FL}(X)$. We begin with a lemma which clearly goes a long way towards limiting the length of covering chains in a free lattice.

LemMA 9.1. Let $w$ be a completely join irreducible element of $\operatorname{FL}(X)$ (with $X$ finite) which has an upper cover. Then $w_{*}$ has no lower cover unless $|X|=3$ and $w$ has the form $x(x y+x z+y z)$ or $(x+y)(x+z)$.

Proof. Let $w \in J(\operatorname{FL}(X))$ have a lower cover $w_{*}$ and an upper cover $v$. Then by the dual of Lemma 8.1, the unique element $w_{1} \in \mathrm{CM}(w)$ such that $w_{1} \neq v$ is totally atomic. Moreover, it is easy to see (as in the proof of Theorem 2.3) that $v=w+$ $\kappa^{\prime}\left(w_{1}\right)$, and so $w=w_{1}\left(w+\kappa^{\prime}\left(w_{1}\right)\right)$.

Suppose $w_{*}$ has a lower cover $u$. Then if $w_{*}$ were meet reducible, $w_{*}$ would be completely join irreducible. Hence by the dual of Corollary $8.3, w$ would be join reducible, contrary to hypothesis. Also $w_{*} \notin X$; indeed, for $x \in X$ we know (by Dean's result from §3) that $x^{*} \succ x \succ x_{*}$ is a maximal covering chain in $\operatorname{FL}(X)$, so we may assume for the duration of this proof that neither $w$ nor $w_{*}$ is a generator. Thus $w_{*}$ must be join reducible, and so completely meet irreducible. Dually to the above, there is a totally atomic $t_{1} \in \mathrm{CJ}\left(w_{*}\right)$ such that $w_{*}=t_{1}+w_{*} \kappa\left(t_{1}\right)$. Our situation now is pictured in Figure 7. Note in particular that $\kappa(w)=w_{*}$, so that when we are done we will have shown that, except near the top and bottom of FL(3), $\kappa(w)=w_{*}$ does not occur when $w$ has an upper cover and $w_{*}$ has a lower cover.

Let us begin by showing that $w_{1}$ and $t_{1}$ cannot be very complex. We will need the following general observation: Let $w \in J(\mathrm{FL}(X))$ have a lower cover. If $w=$ $\Pi_{i}\left(\sum_{j} w_{i j}\right) \Pi_{k} x_{k}$ canonically with $w_{i 1} \nless w$ for each $i$, then $w_{i 1} \leqslant \kappa(w)$, for each $i$. To

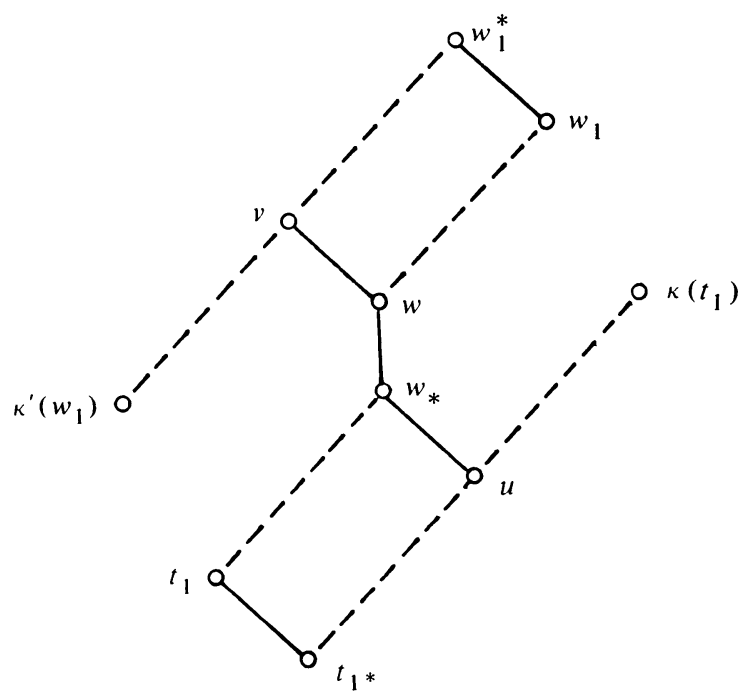

FIGURE 7 
see this, suppose contrarily that, say, $w_{11 *} \nless \kappa(w)$, so that $w \leqslant w_{*}+w_{11 *}$ ( $\leqslant$ $\left.\sum_{j} w_{1 j}\right)$. Applying Whitman's condition (W) to the inclusion

$$
\prod_{i}\left(\sum_{j} w_{i j}\right) \prod_{k} x_{k} \leqslant w_{*}+w_{11 *}
$$

we easily conclude that $\sum_{j} w_{1 j}=w_{*}+w_{11 *}$, contradicting the fact that $w_{11}$ must be a canonical summand of $\sum_{j} w_{1 j}$. Hence $w_{i 1 *} \leqslant \kappa(w)$ for each $i$. For $j>1$, we have even more, viz., $w_{i j} \leqslant w_{*}$ by Theorem 4.4 .

Now in our situation $w_{1}$ is totally atomic and meet irreducible. Hence we have (dually to $(*)$ )

$$
w_{1}=\sigma_{s_{n+1}} \mu_{p_{n}} \cdots \sigma_{s_{2}} \mu_{p_{1}}\left(y_{1}+\cdots+y_{m}\right) .
$$

Assume that, say, $i=1$ is the unique index such that $w_{11}=\sigma_{s_{n+1}} \mu_{p_{n}} \cdots \sigma_{s_{2}} \mu_{p_{1}}\left(y_{1}\right) \nless$ $w$. Then by the above observation $w_{11 *} \leqslant \kappa(w)=w_{*} \leqslant w$, and since $w_{11} \nless w$ we have in fact $w_{11 *} \leqslant w \leqslant \kappa\left(w_{11}\right)$. Now if $n \geqslant 1$ and $s_{2} \neq 0$, then Lemma 8.4 tells us that $\kappa\left(w_{11}\right)$ is not totally atomic. In that case, there are no completely join irreducible elements in the interval $\kappa\left(w_{11}\right) / w_{11 *}$ by Theorem 8.5 ; in particular, we could not have $w_{11 *} \leqslant w \leqslant \kappa\left(w_{11}\right)$. Hence either $n=0$, or $n=1$ and $s_{2}=0$. So we have shown that $w_{1}=\mu_{p}\left(y_{1}+\cdots+y_{m}\right)$, where $p \in \mathscr{P}(X) \cup\{1\}$, and dually $t_{1}=\sigma_{s}\left(z_{1} \cdots z_{k}\right)$, where $s \in \mathscr{S}(X) \cup\{0\}$.

Next suppose $w_{1} \notin X$ and $t_{1} \notin X$, so that $m \geqslant 2$ and $k \geqslant 2$. Now $t_{1} \leqslant w_{*}<w \leqslant$ $w_{1}$, so we may apply $(\mathrm{W})$ to the inclusion

$$
\prod_{i=1}^{k}\left(z_{i}+s\right) \leqslant \sum_{j=1}^{m} y_{j} p .
$$

Thus either $z_{i}+s \leqslant w_{1}$ for some $i$, or $t_{1} \leqslant y_{j} p$ for some $j$, and by duality we may assume the former. If $p \neq 1$ in this case, then we have $z_{i}+s \leqslant w_{1}<p \in \mathscr{P}(X)$, which is impossible. Hence $p=1$ and $w_{1}=y_{1}+\cdots+y_{m}$.

Again we may assume that the indexing is such that $y_{1} \nless w$ and $y_{2}+\cdots+y_{m} \leqslant$ $w_{*}$, and dually $z_{1}+s \neq w_{*}$ while $\left(z_{2}+s\right) \cdots\left(z_{k}+s\right) \geqslant w$. As before this means that $y_{1 *} \leqslant \kappa(w)=w_{*}$ and dually $\left(z_{1}+s\right)^{*} \geqslant \kappa^{\prime}\left(w_{*}\right)=w$. Thus $y_{1 *} \leqslant\left(z_{1}+s\right)^{*}$, i.e.,

$$
y_{1}\left(\sum X-\left\{y_{1}\right\}\right) \leqslant z_{1}+s+\prod\left(X-\left\{z_{1}, s_{1}, \ldots, s_{q}\right\}\right),
$$

where $s=s_{1}+\cdots+s_{q}(q=0$ if $s=0)$. After a few preliminaries we will apply (W) to the inclusion $(\dagger)$.

We claim that

(i) $y_{1} \neq s_{j}$ for any $j$,

(ii) $\left\{y_{1}, z_{1}, s_{1}, \ldots, s_{q}\right\} \subsetneq X$,

(iii) $k$ (the number of $z_{i}$ 's) $=2$ and $y_{1}=z_{2}$.

To prove (i) we note that $y_{1} \leqslant s$ would imply $y_{1} \leqslant \prod_{i=1}^{k}\left(z_{i}+s\right)=t_{1} \leqslant w$, a contradiction. For (ii), if $X=\left\{y_{1}, z_{1}, s_{1}, \ldots, s_{q}\right\}$, then $t_{1}=\left(y_{1}+s\right)\left(z_{1}+s\right)$ and moreover $t_{1} \prec y_{1}+s \prec 1$. This leaves no room for $w$ and $w_{*}$. We will prove (iii) by showing that $y_{1}=z_{i}$ whenever $2 \leqslant i \leqslant k$. So fix $i \geqslant 2$, and note that since $y_{1 *} \leqslant w_{*}$ and $w \leqslant z_{i}+s$, we have $y_{1 *} \leqslant z_{i}+s$. If $s=0$ this clearly implies $y_{1}=z_{i}$ (using 
$|X| \geqslant 3$ ). Otherwise apply (W) to the inclusion $y_{1}\left(\sum X-\left\{y_{1}\right\}\right)<z_{i}+s$. Using (i) and (ii), we easily obtain the desired conclusion $y_{1}=z_{i}$.

Now we return to $(\dagger)$ and apply $(\mathrm{W})$. Once it is observed that

$$
\prod\left(X-\left\{z_{1}, s_{1}, \ldots, s_{q}\right\}\right)
$$

is a meet of at least two variables, it is an easy task, using (i)-(iii), to eliminate all the possibilities. Hence $(\dagger)$ must fail, and we conclude that either $w_{1} \in X$ or $t_{1} \in X$.

By duality, we may as well assume $w_{1}=x \in X$. Recall that $t_{1}=\sigma_{s}\left(z_{1} \cdots z_{k}\right) \leqslant w$. If $s \neq 0$ this implies $s<t_{1} \leqslant w \leqslant x$, a contradiction. Hence $s=0$. Similarly $z_{1} \cdots$ $z_{k} \leqslant x$ implies $x=z_{i}$ for some $i$, and $k \geqslant 2$. So let us assume that $t_{1}=z_{1} \cdots z_{k-1} x$, where $z_{i} \ngtr w_{*}$ and $z_{2} \cdots z_{k-1} x \geqslant w$. Note that since $t_{1} \neq 0,\left\{x, z_{1}, \ldots, z_{k-1}\right\} \varsubsetneqq X$.

Let $B$ denote the lattice in Figure 8. Let $f: \operatorname{FL}(X) \rightarrow B$ be the homomorphism such that $f(x)=a, f\left(z_{1}\right)=b, f\left(z_{i}\right)=1$ for $2 \leqslant i \leqslant k-1$ (this may be vacuous), and $f(y)=c$ for all other $y \in X$.

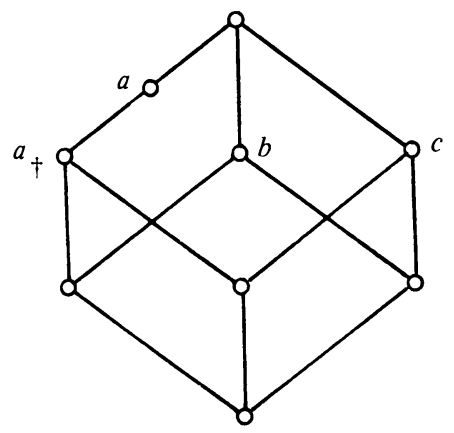

Figure 8

First let us show that $f(w)=a$. Since $z_{1} \ngtr w$ we know that $\Pi\left(X-\left\{z_{1}\right\}\right) \leqslant w$; combining this with previous inequalities we obtain

$$
z_{2} \cdots z_{k-1} x \geqslant w \geqslant z_{1} \cdots z_{k-1} x+\prod\left(X-\left\{z_{1}\right\}\right) .
$$

Applying $f$ to these inclusions shows that $a \geqslant f(w) \geqslant a_{\dagger}$. However, recall that $w=w_{1}\left(w+\kappa^{\prime}\left(w_{1}\right)\right)=x(w+\Pi(X-\{x\}))$. Applying $f$ again now yields $f(w)=a$.

On the other hand,

$$
\begin{aligned}
w_{*} & =t_{1}+w_{*} \kappa\left(t_{1}\right)=t_{1}+w \kappa\left(t_{1}\right) \\
& =z_{1} \cdots z_{k-1} x+w\left(\sum\left(X-\left\{z_{1}, \ldots, z_{k-1}, x\right\}\right)\right) .
\end{aligned}
$$

Applying $f$ to this equation shows that $f\left(w_{*}\right)=a_{+}$.

Therefore $w=\beta_{f}(a)$, and in fact $B \cong L(w)$. Hence we can use the usual algorithm for $\beta$ to find $w$ explictly. Doing this shows that

$$
w=z_{2} \cdots z_{k-1} x\left[z_{1} \cdots z_{k-1} x+z_{2} \cdots z_{k-1} x \prod_{j=1}^{r} y_{j}+z_{1} \cdots z_{k-1} \prod_{j=1}^{r} y_{j}\right],
$$

where $X-\left\{z_{1}, \ldots, z_{k-1}, x\right\}=\left\{y_{1}, \ldots, y_{r}\right\}$. Now we can apply Theorem 4.6 to find $\kappa(w)$. The procedure is straightforward and yields

$$
\kappa(w)=z_{1} x+x\left(\prod_{j=1}^{r} y_{j}\right) .
$$


It remains only to check what restrictions are required in order for $\kappa(w) \leqslant w$ to hold. Clearly we must have $\left\{z_{2}, \ldots, z_{k-1}\right\}=\varnothing$, i.e., $k=1$. Once those terms are removed it is easy to see that we must also have $r=1$ (use (W)). Thus, removing the subscripts, $w=x(x y+x z+y z)$ and $\kappa(w)=x y+x z$ in FL(3). The dual form, with $w=(x+y)(x+z)$ and $\kappa(w)=x+(x+y)(x+z)(y+z)$, is obtained from the case $t_{1} \in X$, and hence the lemma is proved.

We now have all the necessary machinery to complete the proof of our main result on covering chains in free lattices.

THEOREM 9.2. Let $t>u>v>w$ be a covering chain in $\operatorname{FL}(X)$, where $X$ is finite. If $|X|=3$, then

$$
t / w \subseteq(x y+x z+y z) / 0 \cup 1 /(x+y)(x+z)(y+z) .
$$

If $|X| \geqslant 4$, then $w=0$ or $t=1$.

Proof. We know from simple arguments earlier that none of $t, u, v, w$ is a generator. By Lemma 9.1, $u$ is not meet reducible unless

$$
u \in(x y+x z+y z) / 0 \cup 1 /(x+y)(x+z)(y+z)
$$

in $\operatorname{FL}(3)$, and $u$ has an upper cover $t$, so we may assume that $u$ is completely meet irreducible. Dually (or by Corollary 8.3), $v$ is completely join irreducible. By Lemma 8.1 , there is a totally atomic join irreducible element $u_{1} \in \mathrm{CJ}(u)$ such that $u_{1} \nless v$, but $u_{1 *} \leqslant v$. Similarly, there is a totally atomic meet irreducible element $v_{1} \in \mathrm{CM}(v)$ such that $v_{1} \neq u$ although $v_{1}^{*} \geqslant u$. The situation we have just described is diagrammed in Figure 9.

We claim that $v_{1}=\kappa\left(u_{1}\right)$. Indeed, $\kappa\left(u_{1}\right)$ is the unique completely meet irreducible element $q$ of $\operatorname{FL}(X)$ such that $q \ngtr u_{1}$ but $q^{*} \geqslant u_{1}$; hence $v_{1}=\kappa\left(u_{1}\right)$. But since $v_{1}$ is totally atomic, this tells us (by Lemma 8.4) that $u_{1} \in \mathscr{P}(X)$. If $u_{1}=\Pi Y$ with $Y \subseteq X$, then $v_{1}=\sum(X-Y)$.

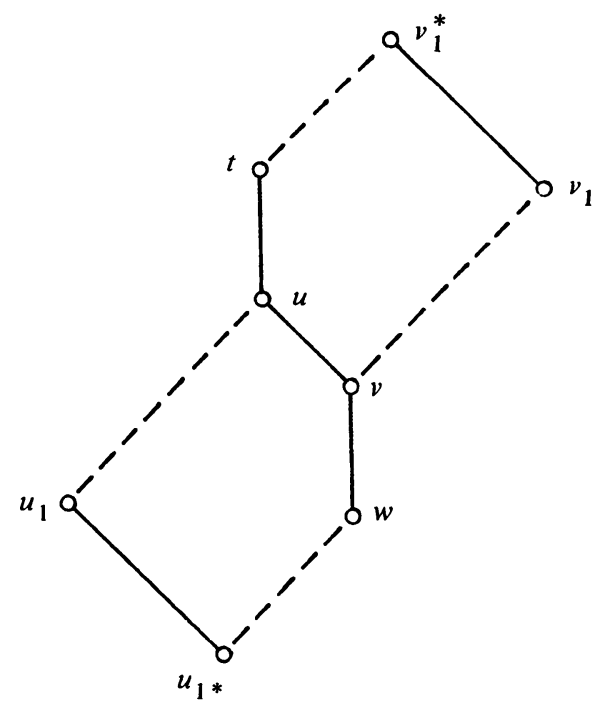

FIGURE 9 
Suppose $u_{1} \notin X$ and $v_{1} \notin X$, i.e., $|Y| \geqslant 2$ and $|X-Y| \geqslant 2$. As $u$ is completely meet irreducible and $u_{1}=\Pi Y \in \mathrm{CJ}(u)$, there is a unique element $y_{1} \in Y$ such that $y_{1} \ngtr u$, and dually there is a unique element $x_{1} \in X-Y$ such that $x_{1} \nless v$. But then $\Pi\left(Y-\left\{y_{1}\right\}\right) \geqslant u \geqslant v \geqslant \sum\left(X-Y-\left\{x_{1}\right\}\right)$, which is a contradiction. So either $u_{1} \in X$ or $v_{1} \in X$.

First let $u_{1}=y \in X$. There is still a unique $x_{1} \in X-\{y\}$ such that $x_{1} \nless v$. Hence $\sum\left(X-\left\{y, x_{1}\right\}\right) \leqslant v$, and thus $\sum\left(X-\left\{x_{1}\right\}\right) \leqslant u_{1}+v=u$. Since $\sum\left(X-\left\{x_{1}\right\}\right) \prec 1$, we conclude that $u=\sum\left(X-\left\{x_{1}\right\}\right)$ and $t=1$.

If $v_{1} \in X$, we obtain dually that $s=0$.

COROllary 9.3. If $w_{0} \succ w_{1} \succ \cdots>w_{n}$ is a maximal covering chain in $\operatorname{FL}(X)(X$ finite) with $w_{0} \neq 1$ and $w_{n} \neq 0$, then $n \leqslant 2$.

10. Finite intervals in $\operatorname{FL}(X)$. In this section we will find all finite intervals $u / v$ in $\operatorname{FL}(X)$. At the top and bottom of $\operatorname{FL}(X)$ there are several finite intervals which are not chains (see Figures 5 and 6). We will show that all other finite intervals in $\operatorname{FL}(X)$ are chains of at most 3 elements.

TheOrem 10.1. Let $u / v$ be a finite interval in $\operatorname{FL}(X)$. Assume that

$$
u / v \nsubseteq \prod_{k \neq i, j}\left(\bar{x}_{i}+\bar{x}_{j}+\bar{x}_{k}\right) / 0
$$

(where $\bar{x}=\Pi(X-\{x\}))$ for each pair $i \neq j$, and dually. Then $u / v$ is a chain and $|u / v| \leqslant 3$.

Proof. It follows from Theorem 9.2 that every finite interval $u / v$ not at the top or bottom of $\mathrm{FL}(X)$ is a lattice of height at most 2. Since $\mathrm{FL}(X)$ is semidistributive, $M_{3}$ is not a sublattice of $\operatorname{FL}(X)$. Hence to prove the theorem it remains only to show that $\mathbf{2} \times \mathbf{2}$ cannot be an interval in $\operatorname{FL}(X)$ except at the extremes. This can be done rather directly, but instead we will give a slightly more involved proof which yields some information about the interval $u / v$ whenever there is a 3-element covering chain $u>w \succ v$.

So let $u \succ w \succ v$ in $\operatorname{FL}(X)$. We may assume by duality that $w$ is join irreducible, so that $v=w_{*}$. Let $w_{1}$ be the totally atomic meet irreducible element of $\mathrm{CM}(w)$ such that $u \nless w_{1}$, whence $u=w+\kappa^{\prime}\left(w_{1}\right)$.

First we observe that if $u>b>w_{*}$ and $b \neq w$, then $u \kappa(w) \geqslant b \geqslant w_{*}+\kappa^{\prime}\left(w_{1}\right)$. Indeed, if $b$ is as given, then $b \ngtr w$ since $u>w$. Combined with $b>w_{*}$, this means $b \leqslant \kappa(w)$. On the other hand, for such a $b, w_{1}>b$ would imply $w=u w_{1} \geqslant b>w_{*}$ whence $b=w$, a contradiction. Thus $w_{1} \neq b$ while $w_{1}^{*} \geqslant u>b$, so $b \geqslant \kappa^{\prime}\left(w_{1}\right)$. The above claim now follows.

In particular, if $\kappa^{\prime}\left(w_{1}\right) \nless \kappa(w)$, then there can be no such element $b$ in $u / w_{*}$, so $u / w_{*}$ is a 3 -element chain. (In this case we can easily show that $u \kappa(w)=w_{*}$ and $w_{*}+\kappa^{\prime}\left(w_{1}\right)=u$.)

However if $\kappa^{\prime}\left(w_{1}\right) \leqslant \kappa(w)$, then $u>u \kappa(w) \geqslant w_{*}+\kappa^{\prime}\left(w_{1}\right)>w_{*}$ since $w \cdot u \kappa(w)$ $=w_{*}$ and $w+\left(w_{*}+\kappa^{\prime}\left(w_{1}\right)\right)=u$. The above remarks then show that $u \succ u \kappa(w) \geqslant$ $w_{*}+\kappa^{\prime}\left(w_{1}\right) \succ w_{*}$ and

$$
u / w_{*}=\left\{u, w, w_{*}\right\} \cup u \kappa(w) /\left(w_{*}+\kappa^{\prime}\left(w_{1}\right)\right) .
$$



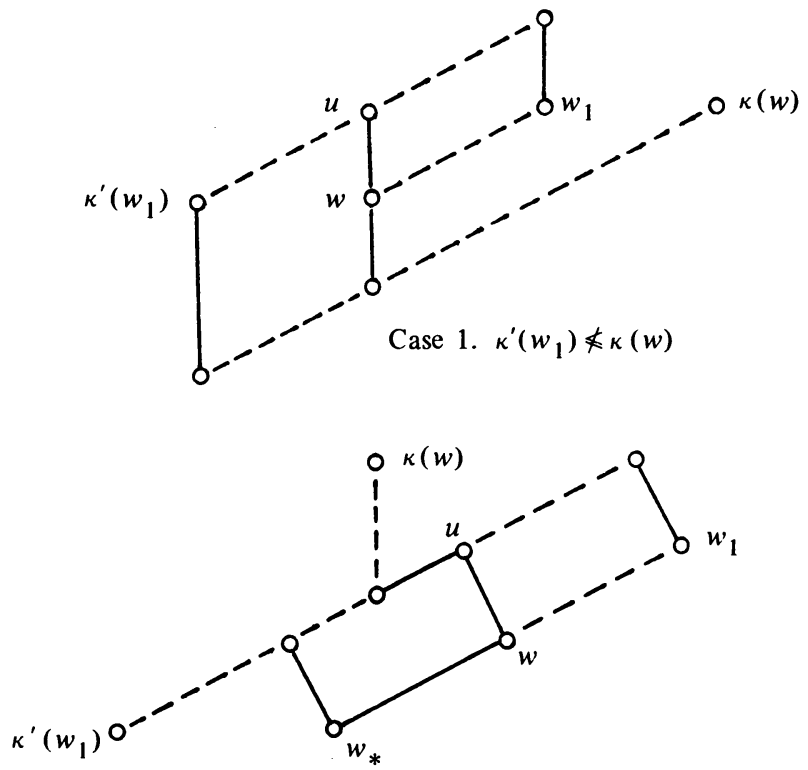

Case 2. $\kappa^{\prime}\left(w_{1}\right) \leqslant \kappa(w)$

FIGURE 10

In particular, the interval $2 \times 2$ occurs only when $u \kappa(w)=w_{*}+\kappa^{\prime}\left(w_{1}\right)$. Supposing this is the case, call this element $v$. Then either $v=\kappa(w)$ (when $v$ is meet irreducible) or $v=\kappa^{\prime}\left(w_{1}\right)$ (in the event that $v$ is join irreducible).

If $v=\kappa(w)$, then $v$ is completely meet irreducible, $v>w_{*}$, and $\kappa^{\prime}\left(w_{1}\right)$ is the unique member of $\mathrm{CJ}(v)$ such that $v \nless w_{*}$ (since $v=w_{*}+\kappa^{\prime}\left(w_{1}\right)$ and $\kappa^{\prime}\left(w_{1}\right)_{*} \leqslant$ $\left.w_{*}\right)$. By Lemma 8.1 this means that $\kappa^{\prime}\left(w_{1}\right)$ is totally atomic, whence by the dual of Lemma 8.4 we have $w_{1} \in \mathscr{S}(X)$.

Let $w_{1}=\sum Y$ with $Y \subseteq X$, so that $\kappa^{\prime}\left(w_{1}\right)=\Pi(X-Y)$. If $|Y| \geqslant 2$ and $|X-Y| \geqslant 2$, then we can argue as in the proof of Theorem 9.2 that there exist $y_{1} \in Y$ and $x_{1} \in \mathrm{X}-Y$ such that $\Sigma\left(Y-\left\{y_{1}\right\}\right) \leqslant w_{*}$ and $\Pi\left(X-Y-\left\{x_{1}\right\}\right) \geqslant v^{*}=u$. Since $w_{*}<u$, this implies $\sum\left(Y-\left\{y_{1}\right\}\right) \leqslant \Pi\left(X-Y-\left\{x_{1}\right\}\right)$, which is impossible. Therefore either $w_{1} \in X$ or $\kappa^{\prime}\left(w_{1}\right) \in X$.

If $w_{1}=y \in X$, we still have that there is an element $x_{1} \in X-\{y\}$ such that $\Pi\left(X-\left\{y, x_{1}\right\}\right) \geqslant u$. In this case $\Pi\left(X-\left\{x_{1}\right\}\right) \geqslant w_{1} u=w$, so we obtain one of the known $2 \times 2$ intervals at the bottom of $\operatorname{FL}(X)$. Dually, if $\kappa^{\prime}\left(w_{1}\right) \in X$ we get that $u / w_{*}$ is one of the known $2 \times 2$ intervals at the top of $\operatorname{FL}(X)$. (In fact, $v=\kappa(w)$ does not hold for the squares at the bottom of $\operatorname{FL}(X)$, and the elements corresponding to $w$ are join reducible in the squares at the top, so $v=\kappa(w)$ never really occurs.)

Now assume that $v=\kappa^{\prime}\left(w_{1}\right)$, whence $v$ is completely join irreducible. Since $u \succ v$, there is a totally atomic meet irreducible element $v_{1} \in \mathrm{CM}(v)$ such that $v_{1} \neq u$. Moreover, $w$ is a completely join irreducible element such that $w \nless v_{1}$ (else $v+w=$ $\left.u \leqslant v_{1}\right)$ but $w_{*} \leqslant v \leqslant v_{1}$, so $\kappa(w)=v_{1}$ and $w=\kappa^{\prime}\left(v_{1}\right)$. 
Thus $w_{1}$ is a canonical meetand of $w=\kappa^{\prime}\left(v_{1}\right)$, and $v_{1}$ is a canonical meetand of $v=\kappa^{\prime}\left(w_{1}\right)$. Now the canonical meetands of $\kappa^{\prime}\left(v_{1}\right)$ are all either generators or else proper joins of the form $\sum A$ where $A \subseteq\left\{\kappa^{\prime}(t): t \in M\left(v_{1}\right)-\left\{v_{1}\right\}\right\}$. Every element $t \in M\left(v_{1}\right)$ is totally atomic since $v_{1}$ is. By the dual of Lemma 8.4, for such an element $\kappa^{\prime}(t)$ is totally atomic if and only if $t \in \mathscr{S}(X)$. If $t=\sum T \in \mathscr{S}(X)$, then $\kappa^{\prime}(t)=\Pi(X-T)$. Now $w_{1}$ is a totally atomic canonical meetand of $\kappa^{\prime}\left(v_{1}\right)$; from the above remarks we conclude that either $w_{1} \in X$ or $w_{1}=\sum A$ with $A \subseteq\left\{\kappa^{\prime}(t)\right.$ : $\left.t \in M\left(v_{1}\right)-\left\{v_{1}\right\}\right\} \cap \mathscr{P}(X)$. The same statement holds with $w_{1}$ and $v_{1}$ interchanged.

If $w_{1}=x \in X$, then $\kappa^{\prime}\left(w_{1}\right)=v=\Pi(X-\{x\})$. Then $v_{1} \in \operatorname{CM}\left(\kappa^{\prime}\left(w_{1}\right)\right)$ implies $v_{1} \in X$ also, say $v_{1}=y$, and $\kappa^{\prime}\left(v_{1}\right)=w=\Pi(X-\{y\})$. In this case $v$ and $w$ are in one of the squares at the bottom of $\operatorname{FL}(X)$, as desired.

In the other case we may assume that both $w_{1}$ and $v_{1}$ are proper joins in $\mathscr{S} \mathscr{P}(X)$. Since $w_{1}$ and $v_{1}$ are both totally atomic, this means that we can write

$$
w_{1}=\mu_{p}\left(x_{1}+\cdots+x_{m}\right) \text { and } v_{1}=\mu_{q}\left(y_{1}+\cdots+y_{n}\right) \text {. }
$$

Then, as in the proof of Lemma 8.4, we calculate that in canonical form

$$
\kappa^{\prime}\left(w_{1}\right)= \begin{cases}\Pi\left(X-\left\{x_{1}, \ldots, x_{m}\right\}\right) & \text { if } p=1 \\ p\left(\bar{p}+\bar{x}_{1}+\cdots+\bar{x}_{m}\right) & \text { if } p \in X \\ \prod_{j=1}^{N}\left(\bar{p}_{j}+\bar{x}_{1}+\cdots+\bar{x}_{m}\right) & \text { if } p=\prod_{j=1}^{N} p_{j} \text { with } N>1\end{cases}
$$

where this time $\bar{x}=\Pi(X-\{x\})$. In no case can we have $v_{1}$ a canonical meetand of $\kappa^{\prime}\left(w_{1}\right)$ (since $m>1$ ), so we conclude that there are no such squares in $\operatorname{FL}(X)$.

The next theorem, although complicated, does give strong information about three-element intervals in free lattices. For example it shows that if the middle element $w$ of a three-element interval $\{u>w \succ v\}$ is join irreducible, then $u$ is a canonical meetand of $w$. This theorem also allows us to show that there are infinicely many three-element intervals in FL(4).

THEOREM 10.2. Let $q$ be a totally atomic meet irreducible element of $\operatorname{FL}(X), X$ finite, and let $q=\sum_{i=1}^{m} q_{i}$ canonically. Let $p_{1}, \ldots, p_{k}$ be completely join irreducible elements satisfying

(1) $\kappa^{\prime}(q)+\sum_{i=1}^{k} p_{i}$ is in canonical form,

(2) $\sum_{i=2}^{m} q_{i} \leqslant \sum_{i=1}^{k} p_{i}<q$

(3) if $k=1, q\left(\kappa^{\prime}(q)+p_{1}\right)>p_{1}$.

Then $w=q\left(\kappa^{\prime}(q)+\sum_{i=1}^{k} p_{i}\right)$ is completely join irreducible and $w$ is the middle element of the three-element interval $\kappa^{\prime}(q)+\sum_{i=1}^{k} p_{i} / w_{*}$. Conversely, if $w$ is join irreducible and the middle element of a three-element interval $u \succ w \succ w_{*}$, and if $q$ is the canonical meetand of $w$ not containing $u$ and $p_{1}, \ldots, p_{k}$ are the canonical summands of $u$ which lie below $w$, then (1), (2) and (3) hold (for the appropriate choice of $q_{1}$ ). Moreover $w=q u=q\left(\kappa^{\prime}(q)+\sum_{i=1}^{k} p_{i}\right)$ canonically.

Proof. First suppose $w$ is join irreducible and the middle element of a threeelement interval $u>w>w_{*}$. Let $q$ be the canonical meetand of $w$ not containing $u$. Then $q$ is totally atomic by the dual of Lemma 8 .1. Since $\kappa^{\prime}(q) \nless w$ and $u / w_{*}$ has 
only three elements we have

$$
\kappa^{\prime}(q)+w_{*}=u \text {. }
$$

Let $w=q w_{2} \cdots w_{n}$ canonically. Then $w=q w_{2} \cdots w_{n} \leqslant \kappa^{\prime}(q)+w_{*}=u$. Now apply (W). If $w \leqslant \kappa^{\prime}(q)$, then $u=\kappa^{\prime}(q)$, which implies that $u$ is completely join irreducible, contradicting the dual of Corollary 8.3. Since $w_{2} \cdots w_{n} \geqslant u$, we must have $w_{2}=u$ and $n=2$. Thus $u$ is a canonical meetand of $w$ and (1) now follows easily from Theorem 4.4, and $w=q\left(\kappa^{\prime}(q)+\sum p_{i}\right)$ canonically also follows.

Write $q=\sum q_{i}$ canonically with $q_{1} \nless w$ but $q_{i} \leqslant w_{*}$ for $i \geqslant 2$ (by 4.4). The canonical expression for $q^{*}$ is $\kappa^{\prime}(q)+\sum q_{i}$. This will follow from Theorem 4.7 once we have $q_{i} \nless \kappa^{\prime}(q)$. But $q$ is totally atomic and so by our description of these elements the automorphisms of $\operatorname{FL}(X)$ fixing $q$ are transitive on the $q_{i}$ 's. Hence if one $q_{i}$ were below $\kappa^{\prime}(q)$ all would be.

Since $w_{*} \geqslant q_{i}, i \geqslant 2, q_{1}+w_{*}=q$. Hence

$$
q^{*}=u+q=\sum p_{i}+\kappa^{\prime}(q)+q=\sum p_{i}+\kappa^{\prime}(q)+q_{1},
$$

as $\sum p_{i}+\kappa^{\prime}(q) \geqslant w_{*}$. Thus $\left\{q_{i}\right\}$ refines $\left\{p_{i}\right\} \cup\left\{\kappa^{\prime}(q), q_{1}\right\}$ from which (2) follows. Since $p_{1} \leqslant w_{*},(3)$ is clear.

To see the other direction let $q$ and $p_{1}, \ldots, p_{k}$ satisfy the hypotheses. Since $q$ is totally atomic the argument above shows that the canonical form of $q^{*}$ is either $\kappa^{\prime}(q)+\sum_{i=1}^{m} q_{i}$ or $\kappa^{\prime}(q)$. However, if $q^{*}=\kappa^{\prime}(q)$, then $\sum p_{i}<q<\kappa^{\prime}(q)$, contradicting (1). Thus $q^{*}=\kappa^{\prime}(q)+\sum_{i=1}^{m} q_{i}$ canonically. Let $w=q\left(\kappa^{\prime}(q)+\sum p_{i}\right)$. We claim that this expression for $w$ is canonical. If $q \leqslant \kappa^{\prime}(q)+\sum p_{i}$, then $\kappa^{\prime}(q)+\sum p_{i}=q^{*}$ by (2) ar d $\kappa^{\prime}(q) \nless q$. Since $q^{*}=\kappa^{\prime}(q)+\sum q_{i}$ canonically, this implies $q_{1} \leqslant \sum p_{i}$. So by (2), $q \leqslant \sum p_{i}<q$, a contradiction. Obviously, $q \ngtr \kappa^{\prime}(q)+\sum p_{i}$. Hence $w$ is meet reducible and thus join irreducible. This, together with (3), implies $w>\sum p_{i}$. Hence $q_{i}<w$ if $i \geqslant 2$. If $w \leqslant q_{1}$, then $q_{2}<w \leqslant q_{1}$, a contradiction (the case $m=1$, i.e., $q$ a generator, is easy). It now follows easily from Whitman's criterion that $w=$ $q\left(\kappa^{\prime}(q)+\sum p_{i}\right)$ canonically.

Once we have shown that $w$ has a lower cover, it will follow that $\kappa^{\prime}(q) \nless \kappa(w)$ because $w \leqslant \kappa^{\prime}(q)+\sum p_{i} \leqslant w_{*}+\kappa^{\prime}(q)$. Also $\kappa^{\prime}(q)+w=\kappa^{\prime}(q)+\sum_{i=1}^{k} p_{i}>w$ as in the proof of Theorem 2.3. Hence, as in the proof of 10.1, the interval $\kappa^{\prime}(q)+$ $\sum_{i=1}^{k} p_{i} / w_{*}$ is a 3-element chain (of course $q$ here corresponas to $w_{1}$ there).

If $w$ failed to have a lower cover, then $L(w)$ would fail to be semidistributive. Since every $u \in J(w)-\{w\}$ has a lower cover, this would mean there exist elements $t_{1}, t_{2} \in L(w)$ such that $w_{\dagger} \leqslant t_{i}$ and $w \nless t_{i}$ for $i=1,2$, but $w \leqslant t_{1}+t_{2}$. Note that for each $i, t_{i} \geqslant w_{\dagger} \geqslant \kappa^{\prime}(q)_{\dagger}$, while $t_{i} \ngtr \kappa^{\prime}(q)$ as $t_{i} \geqslant w_{\dagger} \geqslant \sum_{i=1}^{k} p_{i}$ and $t_{i} \neq w$. Hence each $t_{i} \leqslant \kappa\left(\kappa^{\prime}(q)\right)=q$. Using this when we apply (W) to the inclusion

$$
w=q\left(\kappa^{\prime}(q)+\sum_{i=1}^{k} p_{i}\right) \leqslant t_{1}+t_{2},
$$

we conclude that $q=t_{1}+t_{2}$. Thus $\left\{q_{1}, \ldots, q_{m}\right\}$ refines $\left\{t_{1}, t_{2}\right\}$; in particular, $q_{1} \leqslant t_{i}$ for one of the $t_{i}$ 's. If say $q_{1} \leqslant t_{1}$, then

$$
w \leqslant q=\sum_{j=1}^{m} q_{j} \leqslant q_{1}+\sum_{i=1}^{k} p_{i} \leqslant q_{1}+w_{\dagger} \leqslant t_{1},
$$

a contradiction. Therefore $L(w)$ is semidistributive, and $w$ has a lower cover. 
Let us look at a couple of the types of examples of 3-element intervals we can generate using Theorem 10.2. We begin by choosing a totally atomic meet irreducible element $q$ not of the form $\bar{x}+\bar{y}$. (Since $\kappa^{\prime}(\bar{x}+\bar{y})>\bar{x}+\bar{y}$, (1) and (2) cannot be satisfied with $q=\bar{x}+\bar{y}$.) Then we must choose $p_{1}, \ldots, p_{k}$ satisfying (1), (2) and (3). It is not difficult to show that if also $q \notin X$, then $\left\{p_{1}, \ldots, p_{k}\right\}=\left\{q_{2}, \ldots, q_{m}\right\}$ always works. I.e., if $q$ is a totally atomic meet irreducible element not of the form $x$ or $\bar{x}+\bar{y}$, with $q=\sum_{j=1}^{m} q_{j}$ canonically, then $w=q\left(\kappa^{\prime}(q)+\sum_{j=2}^{m} q_{j}\right)$ is the middle element of a 3-element interval in $\operatorname{FL}(X)$.

A second interesting case arises when we choose $q=t \in X$. If we also let $k=1$, then we are looking for $w=t(\bar{t}+p)$ where $p<t$ must be completely join irreducible and satisfy (1) and (3). It is not hard to show that (3) will be satisfied if and only if $t$ is not a canonical meetand of $p$.

We construct an infinite class of such $p$ 's in $\operatorname{FL}(x, y, z, t)$ as follows: Let $y_{0}=y$, $z_{0}=z, y_{n+1}=y\left(z_{n}+x y\right), z_{n+1}=z\left(y_{n}+x z\right)$ and $w_{n}=\left(y_{n}+x z\right)\left(z_{n}+x y\right)$. At the end of $\S 4$ we showed that each $w_{n}$ is completely join irreducible. Recall that $\mu_{t}$ is the endomorphism of $\operatorname{FL}(x, y, z, t)$ that sends each variable to its meet with $t$. Let $p_{n}=\mu_{t} w_{n}$. For any join irreducible $w$ whose variables do not include $t, J\left(\mu_{t} w\right)=$ $\mu_{t} J(w)$. Hence $L(w) \cong L\left(\mu_{t} w\right)$. Thus each $p_{n}$ is completely join irreducible and clearly $t$ is not a canonical meetand of $p_{n}$. An inductive argument shows that $\bar{t}+p_{n}=x y z+p_{n}$ is in canonical form. Hence, for each $n, w=t\left(\bar{t}+p_{n}\right)$ is the middle element of a three-element interval. Moreover, since $w=t\left(\bar{t}+p_{n}\right)$ canonically, $t\left(\bar{t}+p_{n}\right) \neq t\left(\bar{t}+p_{m}\right)$ if $n \neq m$. Thus FL(4) has infinitely many three-element intervals.

11. Arbitrary intervals in $\operatorname{FL}(X)$. In this chapter we will derive a few simple properties of arbitrary intervals $u / v$ in a free lattice $\operatorname{FL}(X)$. It will no longer be necessary to assume that $X$ is finite.

THEOREM 11.1. If $v \leqslant u$ in $\operatorname{FL}(X)$, then $u / v$ is a projective lattice.

Proof. Necessary and sufficient conditions for a lattice to be projective are given in Theorem 1 of [8]. The only condition which is not immediate to verify is that $\bigcup_{k \geqslant 0} D_{k}(u / v)=u / v$. This, however, is a consequence of the following claim, which is straightforwardly proved by induction. If $w \in D_{k}(\operatorname{FL}(X))$ and $w \leqslant u$, then $v+w \in D_{k}(u / v)$.

On the other hand, a proper infinite interval $u / v$ in $\mathrm{FL}(X)$ cannot be isomorphic to a free lattice. By Day's theorem and the fact that $\operatorname{FL}(X)$ has no coverings when $X$ is infinite, the only possibilities would be for $\operatorname{FL}(m)$ to be isomorphic to a proper interval of $\operatorname{FL}(n)$ with $m$ and $n$ both finite, or for $\operatorname{FL}(X)$ with $X$ infinite to have a proper interval isomorphic (by cardinality arguments) to itself. The former possibility is ruled out by the four-element chains at the top and bottom of $\operatorname{FL}(m)$, along with the observation that the interval from an atom to a coatom of $F L(3)$ is not free. The latter possibility is ruled out because $\operatorname{FL}(X)$ with $X$ infinite has no greatest or least element. (Later in this section we will also show that an infinite "open" interval, $\{w \in \operatorname{FL}(X): v<w<u\}$, in $\operatorname{FL}(X)$ cannot be free.) 
Lemma 11.2. Let $v<u$ in $\operatorname{FL}(X)$ and let $t \in u / v$. Assume $s$ is a canonical joinand of $t$ such that $s$ and $v$ are incomparable. Then $s+v$ is meet and join irreducible in $u / v$.

Proof. Clearly $s+v$ is a proper join and hence meet irreducible.

Note that $s$ is a canonical joinand of $s+v($ in $\operatorname{FL}(X))$. For if $s+v=\sum R$, then $t=\sum R+\sum(\mathrm{CJ}(t)-\{s\})$, whence $s \leqslant r$ for some $r \in R$.

Now let $s+v=\sum Q$ with $Q \subseteq u / v$. Then $s \leqslant q$ for some $q \in Q$; since also $v \leqslant q$ we have $s+v \leqslant q$, whence $s+v=q$. Therefore $s+v$ is join irreducible in $u / v$.

This lemma enables us to show that every interval $u / v$ in a free lattice is generated by the elements which are doubly irreducible in $u / v$. As a convenience of terminology, we will regard $v$ to be doubly irreducible in $u / v$ whenever $v$ is meet irreducible in $u / v$, and dually for $u$.

For $A \subseteq \mathrm{FL}(X)$, let $\langle A\rangle$ denote the sublattice generated by $A$.

Theorem 11.3. Let $v<u$ in $\operatorname{FL}(X)$, and let $D=\{p \in u / v: p$ is join and meet irreducible in $u / v\}$. Then $\langle D\rangle=u / v$.

Proof. With an application in mind, we will prove a slightly stronger statement. Assume $Y \subseteq X$ with $u, v \in\langle Y\rangle$, and let $t \in u / v \cap\langle Y\rangle$. We will show that $t$ $\in\langle D \cap\langle Y\rangle\rangle$. Let $S$ denote $\langle D \cap\langle Y\rangle\rangle$.

Supposing the above statement to be false, let $t$ denote a counterexample of minimal complexity. Surely $t \notin X$, so without loss of generality assume $t=\sum s_{i}$ canonically. Now if $t=v=\sum s_{i}$, then $t$ is meet irreducible in $u / v$, and hence $t \in D \subseteq S$ by our convention. Thus $t \neq v$.

Each $s_{i}$ falls into one of the following three cases.

(1) If $s_{i}>v$, then $s_{i} \in S$ by induction (note $s_{i}<t \leqslant u$ ).

(2) If $s_{i}$ is incomparable with $v$, and $s_{i}+v \in D \cap\langle Y\rangle$ by Lemma 11.2.

(3) If $s_{i}<v$, then case (1) does not occur and case (2) holds for some $j \neq i$ (since $t \neq v)$.

It follows that $t=\sum_{1 \text { holds }} s_{i}+\sum_{2 \text { holds }}\left(s_{i}+v\right) \in S$, contrary to assumption. Hence the original statement is true.

The proof of Theorem 11.3 also shows that no infinite "open" interval $\{w \in$ $\operatorname{FL}(X): v<w<u\}$ in $\operatorname{FL}(X)$ is free. Our previous arguments show that the only possibility would occur when $X$ is infinite and there is a subset $W \subseteq u / v$ with $|W|=|X|$ which freely generates the open interval. Pick $w_{1}, w_{2}, w_{3} \in W$ and let $Y$ be any finite subset of $X$ such that $\left\{u, v, w_{1}, w_{2}, w_{3}\right\} \subseteq\langle Y\rangle$. The proof of Theorem 11.3 then shows that $W \cap\langle Y\rangle$ freely generates the infinite open interval $\{t \in \operatorname{FL}(Y)$ : $v<t<u\}$ in $\operatorname{FL}(Y)$. Since $Y$ is finite, this is ruled out by the earlier arguments using the length of covering chains.

We conclude by mentioning an interesting related problem from Grätzer [10]: Does every infinite interval of $\mathrm{FL}(X)$ contain $\mathrm{FL}(3)$ as a sublattice? Some results about free sublattices of free lattices can be found in [1, 11, 12 and 18]. Also, the reader can easily find conditions on $u$ and $v$ which insure that the projections of the generators $\{u(v+x): x \in X\}$ generate a free sublattice of $u / v$. Nonetheless, at this point we do not even know whether an infinite interval in $\operatorname{FL}(X)$ could be a chain! 


\section{REFERENCES}

1. P. Crawley and R. P. Dilworth, Algebraic theory of lattices, Prentice-Hall, Englewood Cliffs, N. J., 1973.

2. A. Day, A simple solution to the word problem for lattices, Canad. Math. Bull. 13 (1970), 253-254.

3. __ Splitting lattices generate all lattices, Algebra Universalis 7 (1977), 163-169.

4. Characterizations of lattices that are bounded-homomorphic images of sublattices of free lattices, Canad. J. Math. 31 (1979), 69-78.

5. A. Day and J. B. Nation, A note on finite sublattices of free lattices, Algebra Universalis 15 (1982), 90-94.

6. R. A. Dean, Coverings in free lattices, Bull. Amer. Math. Soc. 67 (1961), 548-549.

7. R. Freese, Ideal lattices of lattices, Pacific J. Math. 57 (1975), 125-133.

8. Some order theoretic questions about free lattices and free modular lattices, Proc. Banff Sympos. on Ordered Sets, Reidel, Dordrecht, 1982.

9. R. Freese and J. B. Nation, Projective lattices, Pacific J. Math. 75 (1978), 93-106.

10. G. Grätzer, General lattice theory, Academic Press, New York, 1978.

11. B. Jónsson, Sublattices of a free lattice, Canad. J. Math. 13 (1961), 250́-264.

12. B. Jónsson and J. Kiefer, Finite sublattices of a free lattice, Canad. J. Math. 14 (1962), 487-497.

13. B. Jónsson and J. B. Nation, A report on sublattices of a free lattice, Colloq. Math. Soc. Janos Bolyai, Contributions to Universal Algebra (Szeged), Vol. 17, North-Holland, Amsterdam, 1977, pp. 223-257.

14. R. McKenzie, Equational bases and nonmodular lattice varieties, Trans. Amer. Math. Soc. 174 (1972), $1-43$.

15. J. B. Nation, Bounded finite lattices, Colloq. Math. Soc. Janos Bolyai, Contributions to Universal Algebra (Esztergom), Vol. 29, North-Holland, Amsterdam, 1982, pp. 531-533.

16. Finite sublattices of a free lattice, Trans. Amer. Math. Soc. 269 (1982), 311-337.

17. P. Whitman, Free lattices, Ann. of Math. (2) 42 (1941), 325-330.

18. __ Free lattices. II, Ann. of Math. (2) 43 (1942), 104-115.

19. B. Jónsson, Varieties of lattices: Some open problems, Colloq. Math. Soc. János Bolyai, Contributions to Universal Algebra (Esztergom), Vol. 29, North-Holland, Amsterdam, 1982, pp. 421-436.

Department of Mathematics, University of Hawail, Honolulu, Hawail 96822 